\title{
TRANSFORMATIONS OF WIENER INTEGRALS UNDER A GENERAL CLASS OF LINEAR TRANSFORMATIONS
}

\author{
BY \\ R. H. CAMERON AND W. T. MARTIN
}

Introduction. Let $C$ be the space of all real-valued functions $x(t)$ continuous in $0 \leqq t \leqq 1$, and vanishing at $t=0$. Wiener has defined a measure over the space $C$ and in terms of this measure he has defined an average or integral over $C$ which is intimately related among other things to the theory of the Brownian motion $[1,2]\left({ }^{1}\right)$. The present authors have recently investigated certain aspects of the Wiener integral $[3,4]$ and have obtained for instance in [4] a result which shows how the integral is transformed under translations. In the present paper we determine how the integral transforms under a certain class of linear homogeneous transformations. This result is also combined with the earlier result on translations to yield a transformation formula for the nonhomogeneous transformation-translation plus linear homogeneous transformation. By applying the transformation formula to the special linear transformation

$$
y(t)=x(t)+\lambda \int_{0}^{t} \tan \lambda(s-1) \cdot x(s) d s, \quad-\frac{\pi}{2}<\lambda<\frac{\pi}{2},
$$

we obtain as a corollary the evaluation of the characteristic function of the chance variable $\int_{0}^{1}[x(t)]^{2} d t$; namely,

$$
\int_{C}^{W} \exp \left(i \mu \int_{0}^{1}[x(t)]^{2} d t\right) d_{W} x=\left(\sec (i \mu)^{1 / 2}\right)^{1 / 2} \quad(-\infty<\mu<\infty)
$$

for a suitable determination of the multiple-valued function in the right-hand member.

Our first transformation theorem is as follows:

THEOREM I. Let $K^{1}(t, s)$ be continuous on the closed triangle $[0 \leqq t \leqq s$, $0 \leqq s \leqq 1]$ and let it vanish on the line segment $t=0[0 \leqq s \leqq 1]$; let $K^{2}(t, s)$ be continuous on the closed triangle $[0 \leqq s \leqq t, 0 \leqq t \leqq 1]$; let

$$
K(t, s)= \begin{cases}K^{1}(t, s) \text { when } 0 \leqq t<s, & 0<s \leqq 1 \\ K^{2}(t, s) \text { when } s<t \leqq 1, & 0 \leqq s<1, \\ 2^{-1} K^{1}(s, s)+2^{-1} K^{2}(s, s) \text { when } t=s, & 0 \leqq s \leqq 1\end{cases}
$$

Presented to the Society, April 29, 1944; received by the editors March 16, 1944.

(1) Numbers in brackets refer to the Bibliography at the end of the paper. Other references to the Wiener integral are given in [1] and [2]. 


$$
J(s)=K^{2}(s, s)-K^{1}(s, s),
$$

$0 \leqq s \leqq 1$,

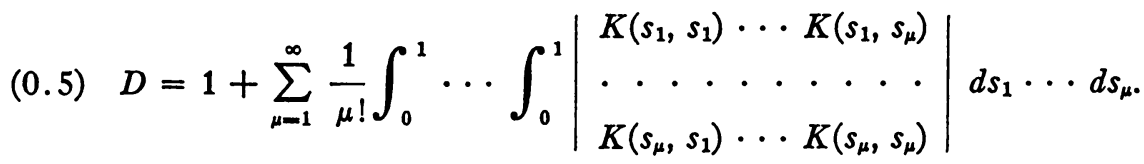

Assume further that $K(t, s)$ is such that the following conditions are satisfied: $(0.6 \mathrm{~A})$ For almost all $s, K(t, s)$ is absolutely continuous in $t$ on $0 \leqq t \leqq 1$ after the jump at $t=s$ is removed by the addition of a step function.

(0.6B) For almost all $s, \partial K(t, s) / \partial t$ is essentially of bounded variation $0 \leqq t \leqq 1$. More precisely, there exists a measurable function $H(t, s)$ which is of bounded variation in $t$ for each $s$ and which for almost all $t, s$ in the square $[0 \leqq t \leqq 1,0 \leqq s \leqq 1]$ is equal to $\partial K(t, s) / \partial t\left(^{2}\right)$.

(0.6C) The function $H(t, s)$ mentioned in $(0.6 \mathrm{~B})$ can be so chosen that

$$
\int_{0}^{1} \sup _{0 \leqq t \leqq 1}|H(t, s)| d s<\infty \quad \text { and } \quad \int_{0}^{1} \operatorname{var}_{0 \leqq t \leqq 1}[H(t, s)] d s<\infty \text {. }
$$

(0.6D) The function $J(s)$ is of bounded variation on $0 \leqq s \leqq 1$.

(0.6E) The determinant $D \neq 0$.

Let $S$ be a Wiener measurable subset of $C$, and let $T S$ be the image of $S$ under

$$
T: \quad y(t)=x(t)+\int_{0}^{1} K(t, \dot{s}) x(s) d s
$$

(so that $y(\cdot) \in T S$ if and only if $y=T x$ and $x(\cdot) \in S$ ). Then we have

$$
\operatorname{meas}_{W}(T S)=|D| \int_{S}^{W} \exp (-\Phi[x]) d_{W} x,
$$

where $\left({ }^{3}\right)$

$$
\begin{aligned}
\Phi[x]= & \int_{0}^{1}\left[\frac{d}{d t} \int_{0}^{1} K(t, s) x(s) d s\right]^{2} d t \\
& +2 \int_{0}^{1}\left[\int_{0}^{1} \frac{\partial}{\partial t} K(t, s) x(s) d s\right] d x(t)+\int_{0}^{1} J(t) d\left\{[x(t)]^{2}\right\} .
\end{aligned}
$$

Moreover if $F[y]$ is any measurable functional for which either member of (0.10) (below) exists, then the other member also exists and the equality holds:

(0.10) $\quad \int_{T S}^{W} F[y] d_{W} y=|D| \int_{S}^{W} F\left[x+\int_{0}^{1} K(\cdot, s) x(s) d s\right] \exp (-\Phi[x]) d_{W} x$.

(2) When we write $\partial K(t, s) / \partial t$ we shall always understand that it is to be replaced, if necessary, by the function $H(t, s)$ to which it is equivalent in the square $[0 \leqq t \leqq 1,0 \leqq s \leqq 1]$.

(3) See footnote 2. 
Remark. It is understood that integrals with a superscript $W$ over the integral sign and a subscript $W$ on the differential are Wiener integrals; ordinary integrals taken with respect to an ordinary real variable are Lebesgue integrals; and ordinary integrals taken with respect to a function of a real variable are Riemann-Stieltjes integrals. The two Stieltjes integrals which occur in (0.9) cannot be put together in the form

$$
2 \int_{0}^{1}\left[\frac{d}{d t} \int_{0}^{1} K(t, s) x(s) d s\right] d x(t)
$$

since $\int_{0}^{1} J(t) d\left\{[x(t)]^{2}\right\}$ cannot be rewritten in the form $2 \int_{0}^{1} J(t) x(t) d x(t)$ and still necessarily exist as a Riemann-Stieltjes integral (see Remark on Lemma 7 of $\S 9)$. Moreover the use of the average of $K^{1}$ and $K^{2}$ on the diagonal in (0.3) is not a mere matter of convenience. It is necessary to obtain the right numerical answer, since the values of $K$ on the diagonal enter very explicitly into the calculation of $D$ in $(0.5)$.

We shall combine this theorem with our theorem on translations given in [4] to yield the following result:

THEOREM II. Let $K(t, s)$ satisfy the hypotheses of Theorem I and let $x_{0}(t)$ be an absolutely continuous function of $C$ whose first derivative $x_{0}^{\prime}(t)$ is essentially of bounded variation on $0 \leqq t \leqq 1$. (By this we mean that there exists a function w $(t)$ of bounded variation on $0 \leqq t \leqq 1$ which equals $x_{0}^{\prime}(t)$ almost everywhere $0 \leqq t \leqq 1$.) Let $S$ be a Wiener measurable subset of $C$ and let $L S$ be the image of $S$ under the transformation

$$
L: \quad y(t)=x(t)+x_{0}(t)+\int_{0}^{1} K(t, s) x(s) d s .
$$

Then

$$
\operatorname{meas}_{W}(L S)=|D| \int_{S}^{W} \exp (-\Psi[x]) d_{W} x
$$

where $\left({ }^{(4)}\right.$

$$
\begin{aligned}
\Psi[x]= & \int_{0}^{1}\left\{\frac{d}{d t}\left[x_{0}(t)+\int_{0}^{1} K(t, s) x(s) d s\right]\right\}^{2} d t \\
& +2 \int_{0}^{1}\left\{\int_{0}^{1} \frac{\partial}{d t}\left[x_{0}(t)+K(t, s) x(s)\right] d s\right\} d x(t) \\
& +\int_{0}^{1} J(s) d\left\{[x(s)]^{2}\right\} .
\end{aligned}
$$

(4) See footnote 2 . 
Moreover if $F[y]$ is any measurable functional for which either member of (0.14) (below) exists, then the other member also exists and they are equal:

$$
\begin{aligned}
& \int_{L S}^{W} F[y] d_{W} y \\
& \quad=|D| \int_{S}^{W} F\left[x+x_{0}+\int_{0}^{1} K(\cdot, s) x(s) d s\right] \exp (-\Psi[x]) d_{W} x .
\end{aligned}
$$

We shall find it simpler to prove first a somewhat less general theorem than Theorem I; namely:

TheOREM Ia. Let $K(t, s)$ satisfy the hypotheses of Theorem I and let $F[x]$ be a bounded functional which is defined on $C$ and is continuous with respect to the uniform topology and vanishes outside a region in $C$ which is bounded in the uniform topology. Then under the transformation $T$ of $(0.7)$ we have

$$
\int_{C}^{W} F[y] d_{W} y=|D| \int_{C}^{W} F\left[x+\int_{0}^{1} K(\cdot, s) x(s) d s\right] \exp (-\Phi[x]) d_{W} x
$$

where $\Phi[x]$ is defined in (0.9).

Most of the present paper will be taken up with the proof of this theorem. Its proof will be based upon several lemmas stated and proved in $\S \S 1$ to 9 . In $\$ 10$ Theorem Ia itself will be proved and in $\$ 11$ Theorem I will be obtained from Theorem Ia by a simple argument. In $\$ 12$ the results of Theorem I and of the translation theorem of [4] will be combined to yield Theorem II. In $\$ 13$ a special case $(0.1)$ of the transformation $T$ will be considered to yield the evaluation of the integral $(0.2)$.

1. Polygonal kernels. In this and the next two sections we consider a linear transformation

$$
T: \quad y(t)=x(t)+\int_{0}^{1} K_{n}(t, s) x(s) d s
$$

where the kernel $K_{n}$ is subject to the following three restrictions:

A. $K_{n}(t, s)$ is continuous in $(t, s)$ in the square $[0 \leqq t \leqq 1,0 \leqq s \leqq 1]$, it vanishes outside the square and on the edge $t=0(0 \leqq s \leqq 1)$ and it is polygonal (of order $n$ ) in $t$ for each $s$. This latter property means that $K_{n}(t, s)$ is linear in $t$ from $j / n$ to $(j+1) / n, j=0,1, \cdots, n-1$.

B. The determinant

$$
\begin{aligned}
\Delta_{n} & =\operatorname{det}\left(A_{j p}+\delta_{j p}\right)_{j, p-1, \ldots, n} \quad\left(\delta_{j p}=\text { Kronecker's delta }\right), \\
A_{j p} & =\int_{(p-1) / n}^{(p+1) / n} K_{n}\left(\frac{j}{n}, s\right)[1-|n s-p|] d s
\end{aligned}
$$

is different from zero: 


$$
\Delta_{n} \neq 0 \text {. }
$$

C. The Fredholm determinant corresponding to the kernel $K_{n}(t, s)$ with $\lambda=-1$ is not zero. (This implies that $T$ is a $1-1$ transformation of $C$ into itself and vice versa, if $T$ is $1-1$, then this Fredholm determinant is different from zero.)

We shall denote by $x_{n}(t)$, corresponding to any function $x(t)$ of $C$, the associated polygonalized function, that is $x_{n}(t)$ is a function which is linear from $j / n$ to $(j+1) / n$ and which is equal to $x(t)$ at the $n+1$ points $p / n, p=0,1$, $\cdots, n$.

Finally, let $F[x]$ be a functional which depends only upon the values of $x$ at the points $t=1 / n, 2 / n, \cdots, n / n$, and is a bounded continuous function of these $n$ values:

$$
F[x]=F_{E}[x(1 / n), x(2 / n), \cdots, x(n / n)]=F\left[x_{n}\right] .
$$

Our purpose is to prove the following lemma.

LEMMA 1.

$$
\begin{aligned}
\int_{C}^{W} F[y] d_{W} y=\left|\Delta_{n}\right| \int_{C}^{W} F\left[x_{n}+\int_{0}^{1} K_{n}(\cdot, s) x_{n}(s) d s\right] \\
\cdot \exp \left\{-\int_{0}^{1}\left[\int_{0}^{1} \frac{\partial}{\partial t} K_{n}(t, s) x_{n}(s) d s\right]^{2} d t\right. \\
\left.-2 \int_{0}^{1}\left[\int_{0}^{1} \frac{\partial}{\partial t} K_{n}(t, s) x_{n}(s) d s\right] d x_{n}(t)\right\} d_{W} x
\end{aligned}
$$

2. Some preliminary results. In this section we shall derive a few preliminary results, preparatory to proving Lemma 1.

First we shall prove:

RESULT 1. If $x_{n}(s)$ is any polygonized function of $C$, then for $j=1, \cdots, n$

$$
\begin{aligned}
\int_{0}^{1} K_{n}\left(\frac{j}{n}, s\right) & x_{n}(s) d s \\
& =\sum_{p=1}^{n} x_{n}\left(\frac{p}{n}\right) \int_{(p-1) / n}^{(p+1) / n} K_{n}\left(\frac{j}{n}, s\right)[1-|n s-p|] d s .
\end{aligned}
$$

Proof. Since $x_{n}(s)$ is polygonal, it has the form

$$
x_{n}(s)=x_{n}((p-1) / n)(p-n s)
$$

$$
+x_{n}(p / n)(n s-p+1) \text { for }(p-1) / n \leqq s \leqq p / n .
$$

Thus 


$$
\begin{aligned}
\int_{0}^{1} K_{n}\left(\frac{j}{n}, s\right) x_{n}(s) d s= & \sum_{p=1}^{n} \int_{(p-1) / n}^{p / n} K_{n}\left(\frac{j}{n}, s\right) x_{n}(s) d s \\
= & \sum_{p=1}^{n} x_{n}\left(\frac{p-1}{n}\right) \int_{(p-1) / n}^{p / n} K_{n}\left(\frac{j}{n}, s\right)(p-n s) d s \\
& +\sum_{p=1}^{n} x_{n}\left(\frac{p}{n}\right) \int_{(p-1) / n}^{p / n} K_{n}\left(\frac{j}{n}, s\right)(n s-p+1) d s \\
= & \sum_{p=0}^{n-1} x_{n}\left(\frac{p}{n}\right) \int_{p / n}^{(p+1) / n} K_{n}\left(\frac{j}{n}, s\right)(p+1-n s) d s \\
& +\sum_{p=1}^{n} x_{n}\left(\frac{p}{n}\right) \int_{(p-1) / n}^{p / n} K_{n}\left(\frac{j}{n}, s\right)(n s-p+1) d s \\
= & \sum_{p=1}^{n} x_{n}\left(\frac{p}{n}\right) \int_{(p-1) / n}^{(p+1) / n} K_{n}\left(\frac{j}{n}, s\right)[1-|n s-p|] d s,
\end{aligned}
$$

where in passing to the final equality we have used the facts that

$$
x_{n}(0)=0, \quad K_{n}(j / n, s)=0 \text { for } 1<s .
$$

RESULT 2. If $x_{n}(s)$ is any polygonalized function of $C$, then

$$
\begin{aligned}
n \sum_{p=1}^{n}\left(A_{j p}-A_{j-1, p}\right) x_{n}\left(\frac{p}{n}\right) & =\int_{0}^{1} \frac{\partial}{\partial t} K_{n}(t, s) x_{n}(s) d s ; \\
(j-1) / n & <t<j / n, \quad j=1, \cdots, n .
\end{aligned}
$$

Proof. By the definition of the $A$ 's (see (1.3)) the left member of (2.5) is equal to

$$
\begin{aligned}
& n \sum_{p=1}^{n} x_{n}\left(\frac{p}{n}\right) \int_{(p-1) / n}^{(p+1) / n}\left[K_{n}\left(\frac{j}{n}, s\right)-K_{n}\left(\frac{j-1}{n}, s\right)\right] \\
& \cdot[1-|n s-p|] d s
\end{aligned}
$$

and by $(2.1)$ this is equal to

$$
n \int_{0}^{1}\left[K_{n}\left(\frac{j}{n}, s\right)-K_{n}\left(\frac{j-1}{n}, s\right)\right] x_{n}(s) d s .
$$

Since $K_{n}(t, s)$ is polygonal of order $n$ in $t$, this is equal to

$$
\int_{0}^{1} \frac{\partial}{\partial t} K_{n}(t, s) x_{n}(s) d s, \quad \frac{j-1}{n}<t<\frac{j}{n} .
$$

This yields (2.5). 
Since the expression (2.5) is independent of $t$ on each interval $(j-1) / n<t$ $<j / n$ and since $x_{n}(s)$ is polygonal of order $n$, the relation (2.5) yields the following result as a corollary.

RESULT 3. For any polygonal function $x_{n}(s)$ of $C$ we have

$$
\begin{aligned}
n \sum_{j=1}^{n}\left[\sum_{p=1}^{n}\left(A_{j p}-A_{j-1, p}\right) x_{n}\left(\frac{p}{n}\right)\right]^{2} & \\
+ & 2 n \sum_{j=1}^{n}\left\{\left[x_{n}\left(\frac{j}{n}\right)-x_{n}\left(\frac{j-1}{n}\right)\right] \sum_{p=1}^{n}\left(A_{j p}-A_{j-1, p}\right) x_{n}\left(\frac{p}{n}\right)\right\} \\
= & \int_{0}^{1}\left[\int_{0}^{1} \frac{\partial}{\partial t} K_{n}(t, s) x_{n}(s) d s\right]^{2} d t \\
+ & 2 \int_{0}^{1}\left[\int_{0}^{1} \frac{\partial}{\partial t} K_{n}(t, s) x_{n}(s) d s\right] d x_{n}(t) .
\end{aligned}
$$

We return to the transformation $T$ of $\S 1$ and associate with it a transformation $T_{E}$ of Euclidean $n$-space defined by

$$
T_{E}: \quad \eta_{j}=\xi_{j}+\sum_{p=1}^{n} A_{j p} \xi_{p}, \quad j=1, \cdots, n .
$$

By property B of $\S 1$ the transformation $T_{E}$ is $1-1$.

3. Proof of Lemma 1. By (1.5) we have

$$
\int_{C}^{W} F[y] d_{W} y=\int_{C}^{W} F_{E}\left[y\left(\frac{1}{n}\right), \cdots, y\left(\frac{n}{n}\right)\right] d_{W} y .
$$

Now the second member depends only upon the values of $y(\cdot)$ at the $n$ points $1 / n, 2 / n, \cdots, n / n$. Hence by Wiener's general theory it can be expressed as the following $n$-fold Lebesgue integral

$$
\begin{aligned}
\frac{n^{n / 2}}{\pi^{n / 2}} \int_{-\infty}^{\infty} \cdots \int_{-\infty}^{\infty} F_{E}\left[\eta_{1}, \cdots, \eta_{n}\right] & \\
& \cdot \exp \left(-n\left[\eta_{1}^{2}+\left(\eta_{2}-\eta_{1}\right)^{2}+\cdots+\left(\eta_{n}-\eta_{n-1}\right)^{2}\right]\right) d \eta_{1} \cdots d \eta_{n} .
\end{aligned}
$$

We now carry out the linear transformation $T_{E}$ defined in (2.10); it carries the $\eta_{j}$ into $\xi_{j}+\sum_{p} A_{j p} \xi_{p}$. Thus (3.2) becomes

$$
\begin{aligned}
\left|\Delta_{n}\right| & \frac{n^{n / 2}}{\pi^{n / 2}} \int_{-\infty}^{\infty} \cdots \int_{-\infty}^{\infty} F_{E}\left[\xi_{1}+\sum_{p=1}^{n} A_{1, p} \xi_{p}, \cdots, \xi_{n}+\sum_{p=1}^{n} A_{n, p} \xi_{p}\right] \\
& \cdot \exp \left\{-n \sum_{j=1}^{n}\left(\xi_{j}-\xi_{j-1}+\sum_{p=1}^{n} A_{j p} \xi_{p}-\sum_{p=1}^{n} A_{j-1, p} \xi_{p}\right)^{2}\right\} d \xi_{1} \cdots d \xi_{n} .
\end{aligned}
$$


In (3.3) $\Delta_{n}$ is the determinant (1.2) of the transformation $T_{E}$. In (3.3) we have also used $\xi$ 's and $A$ 's with subscripts zero with the convention that

$$
\xi_{0}=0 ; \quad A_{0, p}=0, \quad p=1, \cdots, n,
$$

which is consistent with (1.3) and the fact that $K_{n}(0, s)=0$. Now in (3.3) we isolate one part of the exponential function writing (3.3) in the form

$$
\begin{aligned}
\left|\Delta_{n}\right| \frac{n^{n / 2}}{\pi^{n / 2}} \int_{-\infty}^{\infty} \cdots \int_{-\infty}^{\infty} F_{E}\left[\xi_{1}+\sum_{p=1}^{n} A_{1, p} \xi_{p}, \cdots, \xi_{n}+\sum_{p=1}^{n} A_{n, p} \xi_{p}\right] \\
\cdot \exp \left\{-n \sum_{j=1}^{n}\left[\sum_{p=1}^{n}\left(A_{j p}-A_{j-1, p}\right) \xi_{p}\right]^{2}\right. \\
\left.\quad-2 n \sum_{j=1}^{n}\left[\left(\xi_{j}-\xi_{j-1}\right) \sum_{p=1}^{n}\left(A_{j p}-A_{j-1, p}\right) \xi_{p}\right]\right\} \\
\cdot \exp \left\{-n\left[\xi_{1}^{2}+\left(\xi_{2}-\xi_{1}\right)^{2}+\cdots+\left(\xi_{n}-\xi_{n-1}\right)^{2}\right]\right\} d \xi_{1} \cdots d \xi_{n} .
\end{aligned}
$$

By the general theory of Wiener integration this $n$-fold Lebesgue integral is equal to the following Wiener integral:

$$
\begin{aligned}
\left|\Delta_{n}\right| \int_{C}^{W} \exp \left(-2 n \sum_{j=1}^{n}\left\{\left[x\left(\frac{j}{n}\right)-x\left(\frac{j-1}{n}\right)\right] \sum_{p=1}^{n}\left(A_{j p}-A_{j-1, p}\right) x\left(\frac{p}{n}\right)\right\}\right) \\
\cdot \exp \left(-n \sum_{j=1}^{n}\left[\sum_{p=1}^{n}\left(A_{j p}-A_{j-1, p}\right) x\left(\frac{p}{n}\right)\right]^{2}\right) \\
\cdot F_{E}\left[x\left(\frac{1}{n}\right)+\sum_{p=1}^{n} A_{1, p} x\left(\frac{p}{n}\right), \cdots, x\left(\frac{n}{n}\right)\right. \\
\left.+\sum_{p=1}^{n} A_{n, p}\left(\frac{p}{n}\right)\right] d_{W} x .
\end{aligned}
$$

Now we use Result 3 of $\S 2$ together with the simple observation (based on (2.1) and (1.3)) that

$$
\begin{aligned}
F_{E}\left[x\left(\frac{1}{n}\right)+\sum_{p=1}^{n} A_{1, p} x\left(\frac{p}{n}\right), \cdots, x\left(\frac{n}{n}\right)\right. & \left.+\sum_{p=1}^{n} A_{n, p} x\left(\frac{p}{n}\right)\right] \\
& =F\left[x_{n}(\cdot)+\int_{0}^{1} K_{n}(\cdot, s) x_{n}(s) d s\right] .
\end{aligned}
$$

This makes (3.6) equal to the second member of (1.6) and thus Lemma 1 is proved.

4. The Fredholm determinant as a limit. In the next four sections we shall prove the following lemma.

LEMMA 2. Let $K(t, \xi)$ be continuous in the square 


$$
0 \leqq t \leqq 1, \quad 0 \leqq \xi \leqq 1 .
$$

For each positive integer $n$ form the polygonalized function $K_{n}(t, \xi)$ of order $n$ with respect to $t$, namely

$$
\begin{aligned}
& K_{n}(t, \xi)=K((j-1) / n, \xi)(j-n t)+K(j / n, \xi)(n t-j+1), \\
& \quad \text { for } \quad(j-1) / n<t<j / n, \quad j=1, \cdots, n .
\end{aligned}
$$

Form the nth order determinant

$$
\Delta_{n}=\operatorname{det}\left(A_{j k}^{(n)}+\delta_{j k}\right)
$$

where

$$
A_{j k}^{(n)}=\int_{(k-1) / n}^{(k+1) / n} K_{n}\left(\frac{j}{n}, \eta\right)[1-|n \eta-k|] d \eta .
$$

Then

$$
\lim _{n \rightarrow \infty} \Delta_{n}=D
$$

where $D$ is the Fredholm determinant for $K(t, \xi)$ corresponding to $\lambda=-1$ :

$$
D=1+\sum_{\mu=1}^{\infty} \frac{1}{\mu !} \int_{0}^{1} \cdots \int_{0}^{1}\left|\begin{array}{c}
K\left(s_{1}, s_{1}\right) \cdots K\left(s_{1}, s_{\mu}\right) \\
\cdots \\
K\left(s_{\mu}, s_{1}\right) \cdots K\left(s_{\mu}, s_{\mu}\right)
\end{array}\right| d s_{1} \cdots d s_{\mu} .
$$

The proof of this lemma will be made to depend upon two other lemmas which will form the content of the next two sections.

5. The kernel as a limit. In this section we shall prove the following lemma.

LEMMA 3. With each point $(t, \xi)$ of the square (4.1) associate two sequences of positive integers $\left\{j_{n}(t)\right\},\left\{k_{n}(\xi)\right\}$ with

$$
j_{n}(t) \leqq n, \quad k_{n}(\xi) \leqq n,
$$$$
n=1,2, \cdots,
$$

and assume that

$$
\lim _{n \rightarrow \infty} \frac{j_{n}(t)}{n}=t, \quad \text { uniformly in } \quad 0 \leqq t \leqq 1,
$$

and

$$
\lim _{n \rightarrow \infty} \frac{k_{n}(\xi)}{n}=\xi, \text { uniformly in } 0 \leqq \xi \leqq 1 .
$$

Let $K(t, \xi)$ be continuous in the square (4.1) and let $A_{j \mathbf{k}}^{(n)}$ be defined as in (4.4). Then 


$$
\lim _{n \rightarrow \infty} n A_{j_{n}(t), k_{n}(\xi)}^{(n)}=K(t, \xi)
$$

uniformly in (4.1).

Proof. First we observe that

$$
\int_{\left(k_{n}(\xi)-1\right) / n}^{\left(k_{n}(\xi)+1\right) / n}\left(n-n\left|n \eta-k_{n}(\xi)\right|\right) d \eta=1
$$

and the expression $n-n\left|n \eta-k_{n}(\xi)\right|$ is positive in the range considered. Hence

$$
\begin{aligned}
& \left|n A_{j_{n}(t), k_{n}(\xi)}^{(n)}-K(t, \xi)\right| \\
& \quad \leqq \int_{\left(k_{n}(\xi)-1\right) / n}^{\left(k_{n}(\xi)+1\right) / n}\left|K_{n}\left(\frac{j_{n}(t)}{n}, \eta\right)-K(t, \xi)\right|\left(n-n\left|n \eta-k_{n}(\xi)\right|\right) d \eta \\
& \quad \leqq \max \left|K_{n}\left(\frac{j_{n}(t)}{n}, \eta\right)-K(t, \xi)\right|,
\end{aligned}
$$

where the maximum is taken over

$$
0 \leqq t \leqq 1, \quad 0 \leqq \xi \leqq 1, \quad\left(k_{n}(\xi)-1\right) / n \leqq \eta \leqq\left(k_{n}(\xi)+1\right) / n .
$$

We shall show that this maximum approaches zero as $n \rightarrow \infty$. For this purpose let $\epsilon$ be any positive number and write

$$
\begin{aligned}
\left|K_{n}\left(\frac{j_{n}(t)}{n}, \eta\right)-K(t, \xi)\right| \leqq & \left|K\left(\frac{j_{n}(t)}{n}, \eta\right)-K(t, \xi)\right| \\
& +\left|K\left(\frac{j_{n}(t)}{n}, \eta\right)-K_{n}\left(\frac{j_{n}(t)}{n}, \eta\right)\right| .
\end{aligned}
$$

Now since $K(t, \xi)$ is uniformly continuous in (4.1) it follows that there exists a positive number $\delta_{\epsilon}$ such that

$$
\left|K\left(\left(j_{n}(t) / n\right), \eta\right)-K(t, \xi)\right|<\frac{\xi}{2}
$$

for all $n, t, \xi, \eta$ such that

$$
\left|j_{n}(t) / n-t\right|<\delta_{\epsilon}, \quad|\eta-\xi|<\delta_{\epsilon} .
$$

Also since the function $K_{n}(t, \xi)$ of (4.2) converges uniformly to $K(t, \xi)$ when $n$ approaches infinity it follows that there exists a positive number $N !$ such that

$$
\left|K_{n}(t, \xi)-K(t, \xi)\right|<\epsilon / 2 \text { for } n>N !
$$

and all $(t, \xi)$ in $(4.1)$. 
Now let $N_{\mathrm{\epsilon}}$ be so large that the following four conditions are satisfied:

$$
\begin{aligned}
\left|\frac{j_{n}(t)}{n}-t\right| & <\delta_{\epsilon}, \text { for } n>N_{\epsilon}, & 0 \leqq t \leqq 1, \\
\left|\frac{k_{n}(\xi)}{n}-\xi\right| & <\frac{1}{2} \delta_{\epsilon}, \text { for } n>N_{\epsilon}, & 0 \leqq \xi \leqq 1, \\
\frac{1}{N_{\epsilon}}<\frac{1}{2} \delta_{\epsilon}, \quad N ! & N_{\epsilon}<N_{\epsilon \cdot} &
\end{aligned}
$$

By (5.2) and (5.3) such an $N_{\epsilon}$ exists. With this $N_{\epsilon}$ we see by (5.7), (5.8), (5.9), (5.10), and (5.11) that the maximum considered in the final member of (5.6) is less than $\epsilon$ for $n>N_{\epsilon}$. Thus (5.4) holds uniformly and the proof of Lemma 3 is complete.

6. Terms of the Fredholm determinant as limits. In this section we prove the following lemma.

Lemma 4. If $N$ is a fixed positive integer and $q_{1}, \cdots, q_{N}$ a permutation of $1, \cdots, N$, then

$$
\lim _{n \rightarrow \infty} \sum_{p_{1}, \cdots, p_{N}=1}^{n} \prod_{\mu=1}^{N} A_{t_{\mu}, p_{\mu}}^{(n)}=\int_{0}^{1} \cdots \int_{0}^{1} \prod_{\mu=1}^{N} K\left(s_{q_{\mu}}, s_{\mu}\right) d s_{\mu}\left(t_{\mu} \equiv p_{q_{\mu}}\right) .
$$

Proof. The limitand in (6.1) can be written in the form

$$
\int_{0}^{1} \cdots \int_{0}^{1} \Lambda_{n}\left(s_{1}, \cdots, s_{N}\right) d s_{1} \cdots d s_{N}
$$

where $\Lambda_{n}\left(s_{1}, \cdots, s_{N}\right)$ is defined as follows. Let $\left(s_{1}, \cdots, s_{N}\right)$ be any point of the $N$-cube $0 \leqq s_{j} \leqq 1, j=1, \cdots, N$, and for each $j=1, \cdots, N$, let $p_{j}$ be so defined that

$$
\frac{p_{j}-1}{n} \leqq s_{j}<\frac{p_{j}}{n}, \quad j=1, \cdots, N .
$$

Then for this point $\left(s_{1}, \cdots, s_{N}\right)$ the function $\Lambda_{n}$ has the value

$$
n^{N} \prod_{\mu=1}^{N} A_{t_{\mu}, p_{\mu}}^{(n)} \quad\left(t_{\mu} \equiv p_{q_{\mu}}\right) .
$$

For each positive integer $n$ and each point $s$ in $0 \leqq s \leqq 1$ define

$$
\rho_{n}(s)=[n s+1] \text {, }
$$

so that if $\left(s_{1}, \cdots, s_{N}\right)$ lies in (6.3) then

$$
p_{j}=\rho_{n}\left(s_{j}\right) .
$$

Thus $\left[\rho_{n}\left(\dot{s}_{1}\right), \cdots, \rho_{n}\left(s_{N}\right)\right]$ merely names the $N$-cube surrounding the point 
$\left(s_{1}, \cdots, s_{N}\right)$. With these $\rho$ 's we have by Lemma 3

$$
\lim _{n \rightarrow \infty} n A_{\rho_{n}\left(s_{\mu_{\mu}}\right), \rho_{n}\left(s_{\mu}\right)}^{(n)}=K\left(s_{q_{\mu}}, s_{\mu}\right)
$$

uniformly in $0 \leqq s_{q_{\mu}} \leqq 1,0 \leqq s_{\mu} \leqq 1$. Now since $K(t, \xi)$ is bounded in $0 \leqq t \leqq 1$, $0 \leqq \xi \leqq 1$, relation (6.7) implies that there exists an index $N$ such that

$$
n A_{\rho_{n}\left(s q_{\mu}\right), p_{n}\left(s_{\mu}\right)}^{(n)}
$$

is bounded in $0 \leqq s_{\mu} \leqq 1,0 \leqq s_{q_{\mu}} \leqq 1$ for $n>N$. Hence

$$
\begin{aligned}
\lim _{n \rightarrow \infty} \int_{0}^{1} \cdots \int_{0}^{1} \Lambda_{n}\left(s_{1}, \cdots, s_{N}\right) d s_{1} \cdots & d s_{N} \\
& =\int_{0}^{1} \cdots \int_{0}^{1} \prod_{\mu=1}^{N} K\left(s_{q_{\mu}}, s_{\mu}\right) d s_{\mu},
\end{aligned}
$$

and this yields Lemma 4 .

An immediate corollary is the following.

Corollary 1.

$$
\lim _{n \rightarrow \infty} \sum_{p_{1}, \cdots, p_{N}=1}^{n}\left[\operatorname{det}\left(A_{p_{i}, p_{j}}^{(n)}\right)_{i, j=1, \cdots, N}\right]
$$

$$
=\int_{0}^{1} \cdots \int_{0}^{1}\left|\begin{array}{c}
K\left(s_{1}, s_{1}\right) \cdots K\left(s_{1}, s_{N}\right) \\
\cdots \cdot \ldots \cdot \ldots \cdot \cdot \\
K\left(s_{N}, s_{1}\right) \cdots K\left(s_{N}, s_{N}\right)
\end{array}\right| d s_{1} \cdots d s_{N} .
$$

7. Proof of Lemma 2. By (4.4) and the fact that $K_{n}(t, \xi)$ converges uniformly in (4.1) to $K(t, \xi)$ as $n$ approaches infinity we see that

$$
n A_{j k}^{(n)}
$$

is bounded in $n, j, k$. Call its bound $M$. Then by Hadamard's lemma on determinants, the determinant in the first member of (6.10) is in absolute value not greater than

$$
N^{N / 2}(M / n)^{N}
$$

(See for example Whittaker and Watson, Modern analysis, p. 213.) In the limitand in (6.10) there are $n^{N}$ such terms. Hence the limitand in (6.10) in absolute value does not exceed

$$
n^{N} N^{N / 2}(M / n)^{N}=N^{N / 2} M^{N}
$$

and hence the (finite) series 


$$
\begin{aligned}
1+ & \sum_{p=1}^{n} A_{p p}^{(n)}+\frac{1}{2 !} \sum_{p_{1}, p_{2}=1}^{n}\left[\operatorname{det}\left(A_{p_{i}, p_{j}}^{(n)}\right)_{i, j=1,2}\right] \\
& +\cdots+\frac{1}{n !} \sum_{p_{1}, \cdots, p_{n}=1}^{n}\left[\operatorname{det}\left(A_{p_{i}, p_{j}}^{(n)}\right)_{i, j=1}, \cdots, n\right]
\end{aligned}
$$

is dominated (for all $n$ ) by the convergent series

$$
1+\sum_{N=1}^{\infty} \frac{N^{N / 2} M^{N}}{N !}
$$

On using the corollary of the preceding section, we see that (7.4) has as its limit when $n$ approaches infinity the series (4.6).

This yields Lemma 2.

For later purposes we state and prove the following corollary.

CoROLlaRY 2. Let $K_{n}(t, \xi)$ be the polygonalized form of a continuous kernel $K(t, \xi)$ as in equation (4.2) of Lemma 2. Denote by $D$ the Fredholm determinant (4.6) of $K(t, \xi)$ corresponding to the value $\lambda=-1$, and by $D_{n}$ the analogous Fredholm determinant for $K_{n}(t, \xi)$. Then

$$
\lim _{n \rightarrow \infty} D_{n}=D \text {. }
$$

Proof. By (4.2) and the continuity of $K(t, \xi)$ we see first that

$$
\lim _{n \rightarrow \infty} K_{n}(t, \xi)=K(t, \xi) \text { uniformly in (4.1) }
$$

and secondly that there exists a constant $M$ such that

$$
\left|K_{n}(t, \xi)\right|<M \text { for }(t, \xi) \text { in }(4.1) ; \quad n=1,2,3, \cdots .
$$

Hence, for each positive integer $N$,

$$
\begin{aligned}
& \lim _{n \rightarrow \infty} \int_{0}^{1} \cdots \int_{0}^{1}\left|\begin{array}{c}
K_{n}\left(s_{1}, s_{1}\right) \cdots K_{n}\left(s_{1}, s_{N}\right) \\
\cdots \cdot \ldots \cdot \ldots \cdot \ldots \cdot \ldots \\
K_{n}\left(s_{N}, s_{1}\right) \cdots K_{n}\left(s_{N}, s_{N}\right)
\end{array}\right| d s_{1} \cdots d s_{N} \\
& =\int_{0}^{1} \cdots \int_{0}^{1}\left|\begin{array}{c}
K\left(s_{1}, s_{1}\right) \cdots K\left(s_{1}, s_{N}\right) \\
\cdots \cdot \cdots \cdot \cdot \cdot \cdot \cdot \cdot \\
K\left(s_{N}, s_{1}\right) \cdots K\left(s_{N}, s_{N}\right)
\end{array}\right| d s_{1} \cdots d s_{N},
\end{aligned}
$$

and then by Hadamard's theorem on determinants the convergence is bounded, the limitand in (7.9) being bounded by $N^{N / 2} M^{N}$. Hence the series

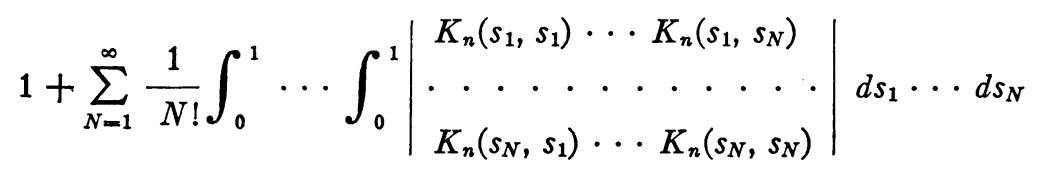


converges boundedly to the series (4.6) for $D$.

This yields Corollary 2.

Similarly we have the following corollary.

Corollary 3. Let $K_{\epsilon}(t, s)$ be Borel measurable in $(t, s)$ for each $\epsilon$ and uniformly bounded for all $t, s, \epsilon$ on $0 \leqq t \leqq 1,0 \leqq s \leqq 1,0<\epsilon<1$, and let $\lim _{\epsilon \rightarrow 0^{+}} K_{\epsilon}(t, s)$ $=K(t, s)$ for all $t, s$ on $0 \leqq t \leqq 1,0 \leqq s \leqq 1$. Then $\lim _{\epsilon \rightarrow 0^{+}} D_{\epsilon}=D$.

8. Preliminary lemmas on Riemann-Stieltjes integrals. In this section and the following section we shall prove three lemmas on Riemann-Stieltjes integrals.

LEMMA 5. Let

$$
f^{n}(t)=n \int_{(p-1) / n}^{p / n} f(s) d s ; \quad \frac{p-1}{n}<t \leqq \frac{p}{n}, \quad p=1, \cdots, n .
$$

Then if $f(s)$ is a function of bounded variation on $0 \leqq s \leqq 1$ and $g(s)$ is continuous on this interval, it follows that

$$
\lim _{n \rightarrow \infty} \int_{0}^{1} f^{n}(t) d g(t)=\int_{0}^{1} f(t) d g(t)
$$

For the proof, we first note that

$$
\begin{aligned}
\int_{0}^{1} f^{n}(t) d g(t) & =\sum_{p=1}^{n} \int_{(p-1) / n}^{p / n} f^{n}(t) d g(t) \\
& =\sum_{p=1}^{n}\left[g\left(\frac{p}{n}\right)-g\left(\frac{p-1}{n}\right)\right] n \int_{(p-1) / n}^{p / n} f(s) d s .
\end{aligned}
$$

Now $n \int_{(p-1) / n}^{p_{n}} f(s) d s$ is the average of $f(s)$ on $(p-1) / n \leqq s \leqq p / n$ and hence there exist two points $s_{r, p}^{\prime}$ and $s_{n, p}^{\prime \prime}$ on this interval such that

$$
f\left(s_{n, p}^{\prime}\right) \leqq n \int_{(p-1) / n}^{p / n} f(s) d s \leqq f\left(s_{n, p}^{\prime \prime}\right) .
$$

Let $\eta_{n, p}^{\prime}=f\left(s_{n, p}^{\prime}\right)$ and $\eta_{n, p}^{\prime \prime}=f\left(s_{n, p}^{\prime \prime}\right)$ if $g(p / n) \geqq g((p-1) / n)$, and let the reverse be true, that is, $\eta_{n, p}^{\prime}=f\left(s_{n, p}^{\prime \prime}\right)$ and $\eta_{n, p}^{\prime \prime}=f\left(s_{n, p}^{\prime}\right)$ if the opposite inequality holds, that is, if $g(k / n)<g((k-1) / n)$. Then we can combine (8.3) and (8.4) and obtain

$$
\begin{aligned}
\sum_{k=1}^{n}\left[g\left(\frac{k}{n}\right)-g\left(\frac{k-1}{n}\right)\right] \eta_{n, k}^{\prime} & \leqq \int_{0}^{1} f^{(n)}(t) d g(t) \\
& \leqq \sum_{k=1}^{n}\left[g\left(\frac{k}{n}\right)-g\left(\frac{k-1}{n}\right)\right] \eta_{n, k .}^{\prime \prime} .
\end{aligned}
$$


But the outer members of (8.5) are Riemann-Stieltjes partial sums for $\int_{0}^{1} f(t) d g(t)$ which exists since $f(t)$ is of B.V. and $g(t)$ is continuous. Hence they approach this integral as $n \rightarrow \infty$, and of course the middle member does also, and the lemma is proved. This lemma enables us to establish the next lemma:

LEMMA 6. Let $H(t, s)$ be measurable in $t$, s on the rectangle $[0 \leqq t \leqq 1,0 \leqq s \leqq 1]$ and satisfy

$$
\int_{0}^{1} M(s) d s<\infty
$$

and

$$
\int_{0}^{1} V(s) d s<\infty
$$

where

$$
M(s)=\sup _{0 \leqq t \leqq 1}|B(t, s)|
$$

and

$$
V(s)=\operatorname{var}_{0 \leqq ı 1}[H(t, s)]
$$

Let

$$
\lim _{n \rightarrow \infty} x_{n}(t)=x(t)
$$

uniformly on $0 \leqq t \leqq 1$, where $x_{n}(t)$ and $x(t)$ are continuous on $0 \leqq t \leqq 1$ and vanish at the origin and satisfy

$$
\left|x_{n}(t)\right| \leqq \mu_{n} \leqq \mu, \quad 0 \leqq t \leqq 1,
$$

and

$$
|x(t)| \leqq \mu, \quad 0 \leqq t \leqq 1
$$

Then if

(8.13) $H^{n}(t, s)=n \int_{(k-1) / n}^{k / n} H(\xi, s) d \xi$ when $\frac{k-1}{n}<t \leqq \frac{k}{n}, k=1,2, \cdots, n$ it follows that

$$
\begin{aligned}
& \lim _{n \rightarrow \infty} \int_{0}^{1}\left[\int_{0}^{1} B^{n}(t, s) x_{n}(s) d s\right]^{2} d t=\int_{0}^{1}\left[\int_{0}^{1} H(t, s) x(s) d s\right]^{2} d t \\
&\left|\int_{0}^{1}\left[\int_{0}^{1} H^{n}(t, s) x_{n}(s) d s\right]^{2} d t\right| \leqq\left[\mu_{n} \int_{0}^{1} M(s) d s\right]^{2},
\end{aligned}
$$


(8.16) $\lim _{n \rightarrow \infty} \int_{0}^{1}\left[\int_{0}^{1} H^{n}(t, s) x_{n}(s) d s\right] d x_{n}(t)=\int_{0}^{1}\left[\int_{0}^{1} H(t, s) x(s) d s\right] d x(t)$,

$$
\left|\int_{0}^{1}\left[\int_{0}^{1} H^{n}(t, s) x_{n}(s) d s\right] d x_{n}(t)\right| \leqq \mu_{n}^{2} \int_{0}^{1}[M(s)+V(s)] d s .
$$

For the proof, we first note that (8.13) implies for almost all $s$

$$
\lim _{n \rightarrow \infty} H^{n}(t, s)=H(t, s)
$$

for almost all $t$, and since by (8.13) and (8.8), (8.11) and (8.12), for all $t$

$$
\begin{gathered}
\left|H^{n}(t, s) x_{n}(s)\right| \leqq \mu_{n} M(s) \leqq \mu M(s), \\
|H(t, s) x(s)| \leqq \mu M(s)
\end{gathered}
$$

we have by $(8.6),(8.13),(8.18),(8.10)$ and the principle of dominated convergence

$$
\begin{aligned}
& \lim _{n \rightarrow \infty} \int_{0}^{1} H^{n}(t, s) x_{n}(s) d s=\int_{0}^{1} H(t, s) x(s) d s, \\
& \lim _{n \rightarrow \infty} \int_{0}^{1} H^{n}(t, s) x(s) d s=\int_{0}^{1} H(t, s) x(s) d s
\end{aligned}
$$

for all $t, 0 \leqq t \leqq 1$. Moreover from (8.19) we have for all $t$

$$
\left|\int_{0}^{1} H^{n}(t, s) x_{n}(s) d s\right| \leqq \mu_{n} \int_{0}^{1} M(s) d s \leqq \mu \int_{0}^{1} M(s) d s,
$$

so that $\int_{0}^{1} H^{n}(t, s) x_{n}(s) d s$ is uniformly bounded in $n$ and $t$. Thus (8.14) follows from (8.21), and (8.15) follows from (8.23).

Next, in order to establish (8.16), we note from (8.13) that

$$
\begin{aligned}
& \int_{0}^{1} H^{n}(t, s) x(s) d s \\
= & n \int_{(k-1) / n}^{k / n} d \xi \int_{0}^{1} H(\xi, s) x(s) d s \text { when } \frac{k-1}{n}<t<\frac{k}{n}, k=1,2, \cdots, n,
\end{aligned}
$$

and that $\int_{0}^{1} H(t, s) x(s) d s$ is of B.V. in $t$ on $0 \leqq t \leqq 1$ since

$$
\begin{aligned}
\int_{0}^{1}\left|d_{t} \int_{0}^{1} H(t, s) x(s) d s\right| & \leqq \int_{0}^{1}\left[|x(s)| \int_{0}^{1}\left|d_{t} H(t, s)\right|\right] d s \\
& \leqq \mu \int_{0}^{1} V(s) d s .
\end{aligned}
$$


Thus by (8.24) and (8.25) it follows from Lemma 5 with $f(t)=\int_{0}^{1} H(t, s) x(s) d s$ that

(8.26) $\lim _{n \rightarrow \infty} \int_{0}^{1}\left[\int_{0}^{1} H^{n}(t, s) x(s) d s\right] d x(t)=\int_{0}^{1}\left[\int_{0}^{1} H(t, s) x(s) d s\right] d x(t)$.

Let us investigate the variation of $H^{n}(t, s)$ in $t$ for fixed $s$. Letting $H^{n}(0, s)$ $=H^{n}(0+0, s)$, we have

$$
\begin{aligned}
\underset{0 \leqq t \leq 1}{\operatorname{var}}\left[H^{n}(t, s)\right] & =\sum_{k=1}^{n-1}\left|H^{n}\left(\frac{k}{n}+0, s\right)-H^{n}\left(\frac{k}{n}-0, s\right)\right| \\
& =\sum_{k=1}^{n-1}\left|n \int_{k / n}^{(k+1) / n} H(t, s) d t-n \int_{(k-1) / n}^{k / n} H(t, s) d t\right| \\
& =\sum_{k=1}^{n-1} n\left|\int_{k / n}^{(k+1) / n}\left[H(t, s)-H\left(t-\frac{1}{n}, s\right)\right] d t\right| \\
& \leqq \sum_{k=1}^{n-1} n \int_{k / n}^{(k+1) / n}\left|H(t, s)-H\left(t-\frac{1}{n}, s\right)\right| d t \\
& =\sum_{k=1}^{n-1} n \int_{0}^{1 / n}\left|H\left(t+\frac{k}{n}, s\right)-H\left(t+\frac{k-1}{n}, s\right)\right| d t \\
& =n \int_{0}^{1 / n}\left\{\sum_{k=1}^{n-1}\left|H\left(t+\frac{k}{n}, s\right)-H\left(t+\frac{k-1}{n}, s\right)\right|\right\} d t \\
& \leqq n \int_{0}^{1}\{\underset{0 \leqq \xi \leqslant 1}{\operatorname{var}} H(\xi, s)\} d t=n \int_{0}^{1 / n} V(s) d t
\end{aligned}
$$

so that for all $s$,

$$
\operatorname{var}_{0 \leqq t \leqq 1}\left[H^{n}(t, s)\right] \leqq V(s)
$$

Thus

(8.28) $\operatorname{var}_{0 \leqq 1 \leqq 1}\left[\int_{0}^{1} H^{n}(t, s) x_{n}(s) d s\right] \leqq \mu_{n} \cdot \int_{0}^{1} V(s) d s \leqq \mu \int_{0}^{1} V(s) d s$

and

$$
\begin{aligned}
\operatorname{var}_{0 \leq t \leq 1}\left\{\int_{0}^{1} H^{n}(t, s)\left[x(s)-x_{n}(s)\right] d s\right\} & \\
& \leqq \max _{0 \leq s \leq 1}\left|x(s)-x_{n}(s)\right| \cdot \int_{0}^{1} V(s) d s .
\end{aligned}
$$

Hence we have from (8.28), (8.29), (8.11), (8.12) 


$$
\begin{aligned}
& \left|\int_{0}^{1} x(t) d_{t} \int_{0}^{1} H^{n}(t, s) x(s) d s-\int_{0}^{1} x_{n}(t) d_{t} \int_{0}^{1} H^{n}(t, s) x_{n}(s) d s\right| \\
& =\mid \int_{0}^{1} x(t) d_{t} \int_{0}^{1} H^{n}(t, s)\left[x(s)-x_{n}(s)\right] d s \\
& +\int_{0}^{1}\left[x(t)-x_{n}(t)\right] d_{t} \int_{0}^{1} H^{n}(t, s) x_{n}(s) d s \\
& \leqq\left[\max _{0 \leqq t \leqq 1}|x(t)|\right] \cdot \operatorname{var}_{0 \leqq t \leqq 1}\left[\int_{0}^{1} H^{n}(t, s)\left[x(s)-x_{n}(s)\right] d s\right] \\
& +\max _{0 \leqq t \leqq 1}\left|x(t)-x_{n}(t)\right| \cdot \operatorname{var}_{0 \leqq t \leqq 1}\left[\int_{0}^{1} H^{n}(t, s) x_{n}(s) d s\right] \\
& \leqq \mu \max _{0 \leqq s \leqq 1}\left|x(s)-x_{n}(s)\right| \cdot \int_{0}^{1} V(s) d s+\max _{0 \leqq t \leqq 1}\left|x(t)-x_{n}(t)\right| \cdot \mu \cdot \int_{0}^{1} V(s) d s ;
\end{aligned}
$$

and since $x_{n}(s) \rightarrow x(s)$ uniformly as $n \rightarrow \infty$, it follows that

$$
\begin{aligned}
\lim _{n \rightarrow \infty}\left\{\int_{0}^{1} x(t) d_{t} \int_{0}^{1} H^{n}(t, s) x(s) d s\right. & \\
& \left.-\int_{0}^{1} x_{n}(t) d_{t} \int_{0}^{1} H^{n}(t, s) x_{n}(s) d s\right\}=0 .
\end{aligned}
$$

But we may integrate these Stieltjes integrals by parts, and when we do we obtain

$$
\begin{aligned}
\int_{0}^{1} & {\left[\int_{0}^{1} H^{n}(t, s) x(s) d s\right] d x(t)-\int_{0}^{1}\left[\int_{0}^{1} H^{n}(t, s) x_{n}(s) d s\right] d x_{n}(s) } \\
= & x(1) \int_{0}^{1} H^{n}(1, s) x(s) d s-x_{n}(1) \int_{0}^{1} H^{n}(1, s) x_{n}(s) d s \\
& -\int_{0}^{1} x(t) d_{t} \int_{0}^{1} H^{n}(t, s) x(s) d s+\int_{0}^{1} x_{n}(t) d_{t} \int_{0}^{1} H^{n}(t, s) x_{n}(s) d s
\end{aligned}
$$

and (8.31), (8.30), (8.21), (8.22) imply

$$
\begin{aligned}
\lim _{n \rightarrow \infty}\left\{\int_{0}^{1}\left[\int_{0}^{1} H^{n}(t, s) x(s) d s\right] d x(t)\right. & \\
& \left.-\int_{0}^{1}\left[\int_{0}^{1} H^{n}(t, s) x_{n}(s) d s\right] d x_{n}(t)\right\}=0,
\end{aligned}
$$

which with (8.26) implies our third result, namely, (8.16).

Finally, to establish (8.17) we integrate the left member by parts and use (8.11), (8.23) and (8.28), obtaining 


$$
\begin{aligned}
\left|\int_{0}^{1}\left[\int_{0}^{1} H^{n}(t, s) x_{n}(s) d s\right] d x_{n}(t)\right| \\
=\left|x_{n}(1) \int_{0}^{1} H^{n}(1 ; s) x_{n}(s) d s-\int_{0}^{1} x_{n}(t) d_{t} \int_{0}^{1} H^{n}(t, s) x_{n}(s) d s\right| \\
\leqq \mu_{n} \cdot\left(\mu_{n} \int_{0}^{1} M(s) d s\right)+\mu_{n} \cdot\left(\mu_{n} \int_{0}^{1} V(s) d s\right)
\end{aligned}
$$

which establishes (8.17) and completes the proof of the lemma.

9. Preliminary lemma on the jump of the kernel. In order to remove discontinuities from the kernel of the transformation with which we shall deal in our theorems, we introduce an odd function which has a jump at the origin and vanishes outside the $\epsilon$-neighborhood of the origin, namely

$$
\Omega_{\epsilon}(s)=\left\{\begin{array}{ccc}
\operatorname{sgn} s-s / \epsilon & \text { when } & |s| \leqq \epsilon \\
0 & \text { when } & |s|>\epsilon
\end{array}\right.
$$

This function, which is defined for all positive $\epsilon$ and all real $s$, will be multiplied by one-half of the amount of the jump of the discontinuous kernel and then subtracted from it to produce a continuous kernel. The limits will be taken as $\epsilon \rightarrow 0^{+}$, and in particular the limit given in the following lemma will play an important role:

LEMMA 7. Let $J(s)$ be continuous and of bounded variation on $0 \leqq s \leqq 1$ and let $x(s)$ be continuous on this interval and vanish at $s=0$. Then if for $0<\epsilon<1 / 2$ $\Omega_{\epsilon}(s)$ is defined by $(9.1)$, we have

$$
\lim _{\epsilon \rightarrow 0^{+}} \int_{0}^{1}\left[\int_{0}^{1} \frac{\partial}{\partial t} \Omega_{\epsilon}(s-t) J(s) x(s) d s\right] d x(t)=\int_{0}^{1} J(t) d\left\{[x(t)]^{2}\right\} .
$$

Moreover, if

$$
\begin{aligned}
& |x(s)| \leqq \mu, \\
& 0 \leqq s \leqq 1 \text {, } \\
& V=\operatorname{var}_{0 \leqq s \leqq 1}[J(s)] \text {, } \\
& M=\max _{0 \leqq s \leqq 1}|J(s)|,
\end{aligned}
$$

we have

$$
\left|\int_{0}^{1}\left[\int_{0}^{1} \frac{\partial}{\partial t} \Omega_{e}(s-t) J(s) x(s) d s\right] d x(t)\right| \leqq \mu^{2}(M+V) .
$$

Remark. This lemma is the critical lemma for the "Volterra case" of our theorems, that is, the case in which the kernel of the transformation has a line of jumps along the diagonal; and this lemma explains why the Stieltjes 
integral of $J$ in our theorems is written as it is. We could not, for instance, replace the right member of (9.2) by

$$
2 \int_{0}^{1} J(t) x(t) d x(t)
$$

which appears to be formally equivalent to it, because this integral need not exist as a Riemann-Stieltjes integral. Thus, if $J(t) \equiv 1$ and $x(t)=t^{1 / 2} \cos \pi / t$ when $t \neq 0$, the R-S sums of (9.7) would be made arbitrarily great, so that (9.7) does not exist; but of course the right member of (9.2) would exist and would have the value unity. When (9.7) fails to exist in the R-S sense, we may think of the right member of (9.2) as giving a sort of "Cauchy principal value" or "Cesàro mean," and the fact that we have to use a "Cauchy principal value" here is connected in some indirect and deep lying way with the fact that in our theorems we have to use an arithmetic mean along the diagonal of the kernel in calculating the Fredholm determinant. Moreover the latter fact is not a mere matter of normalization, but has definite significance in the numerical calculation of our results. It might seem, therefore, that the type of Stieltjes integral used here, or the type of average used in evaluating it, may not be without numerical significance in the results. At any rate, we can prove our theorems using an integral of the type used in (9.2), and have so far not been able to do so with any other type.

In order to facilitate the proof of the lemma, we first extend the definition of $x(s)$ and $J(s)$ so that they are continuous and constant outside the interval $0 \leqq s \leqq 1$. In particular,

$$
x(s)=0 \text { when } s \leqq 0 .
$$

Now in order to evaluate the repeated integral in (9.2), we first note by direct calculation that

$$
\frac{\partial}{\partial t} \Omega_{\epsilon}(s-t)=\left\{\begin{array}{lll}
1 / \epsilon & \text { when } & |s-t|<\epsilon, s \neq t \\
0 & \text { when } & |s-t|>\epsilon,
\end{array}\right.
$$

and since the non-existence of the function at the three points $s=t-\epsilon, s=t$, $s=t+\epsilon$ does not affect the Lebesgue integral, we obtain by using (9.8), (9.9), and integration by parts

$$
\begin{aligned}
\int_{0}^{1} & {\left[\int_{0}^{1} \frac{\partial}{\partial t} \Omega_{\epsilon}(s-t) J(s) x(s) d s\right] d x(t) } \\
& =\frac{1}{\epsilon} \int_{0}^{1}\left[\int_{t-\epsilon}^{\min (t+\epsilon, 1)} J(s) x(s) d s\right] d x(t) \\
& =\frac{1}{\epsilon} x(1) \int_{1-\epsilon}^{1} J(s) x(s) d s-\frac{1}{\epsilon} \int_{0}^{1} x(t) d_{t} \int_{t-\epsilon}^{\min (t+\epsilon, 1)} J(s) x(s) d s
\end{aligned}
$$


Defining the smoothing function

$$
J_{\epsilon}(t)=\frac{1}{\epsilon} \int_{t}^{t+\epsilon} J(\xi) d \xi,
$$

we are able to simplify the last integral, thus

$$
\begin{aligned}
\frac{1}{\epsilon} \int_{0}^{1} x(t) d \int_{t-\epsilon}^{\min (t+\epsilon, 1)} J(s) x(s) d s \\
=\frac{1}{\epsilon} \int_{0}^{1} x(t)\left[\frac{d}{d t} \int_{t-\epsilon}^{\min (t+\epsilon, 1)} J(s) x(s) d s\right] d t \\
=\frac{1}{\epsilon}\left[\int_{0}^{1-\epsilon} x(t) J(t+\epsilon) x(t+\epsilon) d t-\int_{0}^{1} x(t) J(t-\epsilon) x(t-\epsilon) d t\right] \\
=\frac{1}{\epsilon}\left[\int_{0}^{1-\epsilon} x(t) J(t+\epsilon) x(t+\epsilon) d t-\int_{0}^{1-\epsilon} x(t+\epsilon) J(t) x(t) d t\right] \\
=\frac{1}{\epsilon} \int_{0}^{1-\epsilon} x(t) x(t+\epsilon)[J(t+\epsilon)-J(t)] d t \\
=\frac{1}{\epsilon} \int_{0}^{1} x(t) x(t+\epsilon)[J(t+\epsilon)-J(t)] d t \\
\quad-\frac{1}{\epsilon} \int_{1-\epsilon}^{1} x(t) x(t+\epsilon)[J(t+\epsilon)-J(t)] d t \\
=\int_{0}^{1} x(t) x(t+\epsilon)\left[\frac{d}{d t} J_{\epsilon}(t)\right] d t-x\left(\tau_{\epsilon}\right) x\left(\tau_{\epsilon}+\epsilon\right)\left[J\left(\tau_{\epsilon}+\epsilon\right)-J\left(\tau_{c}\right)\right]
\end{aligned}
$$

where $1-\epsilon \leqq \tau_{\epsilon} \leqq 1$, the existence of $\tau_{\epsilon}$ being established by the law of the mean for integrals. Hence, if we can show that

$$
\lim _{\epsilon \rightarrow 0^{+}} \int_{0}^{1} x(t) x(t+\epsilon)\left[\frac{d}{d t} J_{\epsilon}(t)\right] d t=\int_{0}^{1}[x(t)]^{2} d J(t),
$$

it will follow that

$$
\lim _{\epsilon \rightarrow 0^{+}} \frac{1}{\epsilon} \int_{0}^{1} x(t) d_{t} \int_{t \rightarrow \epsilon}^{\min (t+\epsilon, 1)} J(s) x(s) d s=\int_{0}^{1}[x(t)]^{2} d J(t),
$$

since $\lim _{\epsilon \rightarrow 0+}\left[J\left(\tau_{\epsilon}+\epsilon\right)-J\left(\tau_{\epsilon}\right)\right]=J(1)-J(1)=0$ and $x(t)$ is bounded.

In order to establish (9.13), let $J(t)=P(t)-Q(t)$ where $P(t)$ and $Q(t)$ are continuous everywhere and are increasing (not merely monotonically increasing) functions on the interval $0 \leqq t \leqq 1$ and are constant outside that interval. Then if 


$$
P_{\bullet}(t)=\frac{1}{\epsilon} \int_{t}^{t+c} P(\xi) d \xi \text { and } Q_{\epsilon}(t)=\frac{1}{\epsilon} \int_{t}^{t+\epsilon} Q(\xi) d \xi \text {, }
$$

we note that $P_{\epsilon}(t)$ and $Q_{\epsilon}(t)$ are (definitely) increasing functions on the interval $-\epsilon \leqq t \leqq 1$ and constant elsewhere, that $J_{\epsilon}(t)=P_{c}(t)-Q_{\epsilon}($.$) everywhere,$ and that $P_{\epsilon}(-\epsilon)=P(0), Q_{\epsilon}(-\epsilon)=Q(0), P_{\epsilon}(1)=P(1), Q_{\epsilon}(1)=Q(1)$. Now $P_{\epsilon}(t)$ and $P(t)$ have continuous increasing inverses $P_{\epsilon}^{-1}(s)$ and $P^{-1}(s)$ defined on $P(0) \leqq s \leqq P(1)$, and $Q_{\epsilon}(t)$ and $Q(t)$ have inverses $Q_{\epsilon}^{-1}(s)$ and $Q^{-1}(s)$ on $Q(0) \leqq s \leqq Q(1)$. Since $\lim _{\epsilon \rightarrow 0+} P_{\epsilon}(t)=P(t)$ uniformly in $t$, we have $\lim _{\epsilon \rightarrow 0+} P_{\epsilon}^{-1}(s)$ $=P^{-1}(s)$ for all $s$ on $P(0) \leqq s \leqq P(1)$, and hence

$$
\begin{aligned}
\lim _{\epsilon \rightarrow 0^{+}} \int_{0}^{1} x(t) x(t+\epsilon) & {\left[\frac{d}{d t} P_{\epsilon}(t)\right] d t=\lim _{\epsilon \rightarrow 0^{+}} \int_{0}^{1} x(t) x(t+\epsilon) d P_{\epsilon}(t) } \\
& =\lim _{\epsilon \rightarrow 0^{+}} \int_{P(0)}^{P(1)} x\left[P_{\epsilon}^{-1}(s)\right] x\left[P_{\epsilon}^{-1}(s)+\epsilon\right] d s \\
& =\int_{P(0)}^{P(1)} x\left[P^{-1}(s)\right] x\left[P^{-1}(s)\right] d s \\
& =\int_{0}^{1}[x(t)]^{2} d P(t) .
\end{aligned}
$$

Similarly

$$
\lim _{\epsilon \rightarrow 0^{+}} \int_{0}^{1} x(t) x(t+\epsilon)\left[\frac{d}{d t} Q_{\epsilon}(t)\right] d t=\int_{0}^{1}[x(t)]^{2} d Q(t),
$$

and since $J(t)=P(t)-Q(t)$ and $J_{c}(t)=P_{c}(t)-Q_{e}(t)$ we obtain $(9.13)$ by subtracting (9.17) from (9.16), and (9.13) establishes (9.14) as noted above. Now taking limits in (9.10), integrating by parts, and applying (9.14), we have

$$
\begin{aligned}
& \lim _{\epsilon \rightarrow 0^{+}} \int_{0}^{1}\left[\int_{0}^{1} \frac{\partial}{\partial t} \Omega_{\epsilon}(s-t) J(s) x(s) d s\right] d x(t) \\
& =\lim _{\rightarrow \rightarrow 0^{+}}\left[x(1) \cdot \frac{1}{\epsilon} \int_{1-\epsilon}^{1} J(s) x(s) d s\right]-\lim _{\rightarrow \rightarrow 0^{+}} \frac{1}{\epsilon} \int_{0}^{1} x(t) d_{t} \int_{t \rightarrow-}^{\min (t+\epsilon, 1)} J(s) x(s) d s \\
& =x(1) J(1) x(1)-\int_{0}^{1}[x(t)]^{2} d J(t) \\
& =\int_{0}^{1} J(t) d[x(t)]^{2},
\end{aligned}
$$

and this establishes (9.2).

To establish (9.6), let 


$$
\begin{aligned}
& P^{*}(t)=\frac{J(t)}{2}+\frac{1}{2} \operatorname{var}_{-\infty<\xi \leqq t}[J(\xi)], \\
& Q^{*}(t)=-\frac{J(t)}{2}+\frac{1}{2} \operatorname{var}_{-\infty<\xi \leqq t}[J(\xi)], \\
& P_{\epsilon}^{*}(t)=\frac{1}{\epsilon} \int_{t}^{t+\epsilon} P^{*}(\xi) d \xi,
\end{aligned}
$$

and

$$
Q_{*}^{*}(t)=\frac{1}{\epsilon} \int_{t}^{t+\epsilon} Q^{*}(\xi) d \xi
$$

and note that $P^{*}, Q^{*}, P_{\epsilon}^{*}, Q_{\epsilon}^{*}$ are monotonically increasing functions for which

$$
\underset{0 \leqq t \leqq 1}{\operatorname{var}}\left[P^{*}(t)\right]=P^{*}(1)-P^{*}(0)=P_{\epsilon}^{*}(1)-P_{\epsilon}^{*}(-\epsilon)=\operatorname{var}_{-\epsilon \leqq t \leqq 1}\left[P_{\epsilon}^{*}(t)\right]
$$

and

$$
\underset{0 \leqq t \leqq 1}{\operatorname{var}}\left[Q^{*}(t)\right]=Q^{*}(1)-Q^{*}(0)=Q_{\epsilon}^{*}(1)-Q_{\epsilon}^{*}(-\epsilon)=\operatorname{var}_{-\epsilon \leqq t \leqq 1}\left[Q_{\epsilon}^{*}(t)\right] .
$$

Now $J(t)=P^{*}(t)-Q^{*}(t)$ and $J_{c}(t)=P_{c}^{*}(t)-Q_{c}^{*}(t)$, and we have

$$
\begin{aligned}
\operatorname{var}_{-\bullet \leqq t \leqq 1}\left[J_{c}(t)\right] & \leqq \operatorname{var}_{-\bullet \leqq t \leqq 1}\left[P_{c}^{*}(t)\right]+\operatorname{var}_{-\iota \leqq t \leqq 1}\left[Q_{\epsilon}^{*}(t)\right] \\
& =P^{*}(1)-P^{*}(0)+Q^{*}(1)-Q^{*}(0)=\operatorname{var}_{0 \leqq t \leqq 1}[J(t)]=V .
\end{aligned}
$$

Then from (9.12), (9.3), and (9.18) we see that

$$
\left|\frac{1}{\epsilon} \int_{0}^{1} x(t) d_{t} \int_{t \rightarrow}^{\min (t+\epsilon, 1)} J(s) x(s) d s\right|
$$

$$
\begin{aligned}
& =\left|\frac{1}{\epsilon} \int_{0}^{1-\epsilon} x(t) \dot{x}(t+\epsilon)[J(t+\epsilon)-J(t)] d t\right| \\
& =\left|\int_{0}^{1-\epsilon} x(t) x(t+\epsilon) d J_{\epsilon}(t)\right| \leqq \mu^{2} V,
\end{aligned}
$$

and since

$$
\left|x(1) \frac{1}{\epsilon} \int_{1-\epsilon}^{1} J(s) x(s) d s\right| \leqq \mu \cdot M \cdot \mu
$$

we obtain (9.6) from (9.10), (9.19), and (9.20). This completes the proof of the lemma.

10. Proof of Theorem Ia. To prove Theorem Ia we approximate $K(t, s)$ 
by kernels of the type which appears in Lemma 1 . Thus, for $0<\epsilon<1 / 2$, let

$$
\Lambda_{\epsilon}(t, s)=\frac{1}{2}\left[\Omega_{\epsilon}(s-t)-\Omega_{\epsilon}(s+0)\right] J(s) \quad \text { on } \quad\left[\begin{array}{l}
0 \leqq t \leqq 1 \\
0 \leqq s \leqq 1
\end{array}\right]
$$

where $\Omega_{\epsilon}(s)$ is defined by $(9.1)$; and let

$$
K_{\epsilon}(t, s)=K(t, s)+\Lambda_{\epsilon}(t, s) .
$$

It is clear that $K_{\epsilon}(t, s)$ is continuous in the whole square $[0 \leqq t \leqq 1,0 \leqq s \leqq 1]$ since both $K$ and $\Lambda_{\epsilon}$ are continuous except when $t=s$, and for this diagonal, as $t$ increases (for fixed $s$ ) and passes $t=s$, the functions $K$ and $\Lambda_{\mathbf{c}}$ change by $J(s)$ and $-J(s)$ respectively. Moreover for each of these functions, the value on the diagonal is the arithmetic mean of the right-hand and left-hand limits. We also note that $K_{\epsilon}(t, s)$ is uniformly bounded, that $K_{\epsilon}(0, s)=0(0 \leqq s \leqq 1)$, and that for all $t, s$ in the square (including the diagonal)

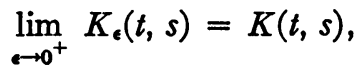

since for all $s$ (including $s=0$ )

$$
\lim _{\rightarrow \rightarrow 0^{+}} \Omega_{e}(s)=0 .
$$

Next, for each positive integer $n$ form the polygonalized function $K_{\epsilon, n}(t, s)$ of order $n$ with respect to $t$, namely,

$$
\begin{aligned}
K_{\mathrm{c}, n}(t, s)= & K_{\mathrm{e}}\left(\frac{j-1}{n}, s\right)(j-n t)+K_{\mathrm{e}}\left(\frac{j}{n}, s\right)(n t-j+1) \\
& \quad \text { for } \frac{j-1}{n} \leqq t \leqq \frac{j}{n} ; j=1,2, \cdots, n ; \quad 0 \leqq s \leqq 1 .
\end{aligned}
$$

We note that $K_{\epsilon, n}(0, s)=0$ for $0 \leqq s \leqq 1$. Let $D_{\epsilon}$ and $D_{\epsilon, n}$ denote the Fredholm determinants obtained by putting $K_{\epsilon}$ and $K_{\epsilon, n}$ in place of $K$ in (0.5). Then, since $K_{\epsilon, n}$ and $K_{\epsilon}$ are obviously bounded, we have by (10.3), (10.4), and Corollaries 2 and 3 of Lemma 2,

$$
\lim _{\epsilon \rightarrow 0^{+}} D_{\epsilon}=D
$$

and

$$
\lim _{n \rightarrow \infty} D_{\epsilon, n}=D_{\epsilon} .
$$

Thus, since $D \neq 0$ by $(0.6 \mathrm{E})$, there exists an $\epsilon_{1}$ such that $D_{\epsilon} \neq 0$ when $0<\epsilon<\epsilon_{1}$. Similarly, there exists $n=n_{1}(\epsilon)$ such that $D_{\epsilon, n} \neq 0$ when $n>n_{1}(\epsilon)$ and $0<\epsilon<\epsilon_{1}$. Moreover if $\Delta_{\epsilon, n}$ is obtained by replacing $K$ by $K_{\epsilon}$ (and hence $K_{n}$ by $K_{\epsilon, n}$ ) in Lemma 2, we have 


$$
\lim _{n \rightarrow \infty} \Delta_{\epsilon, n}=D_{e}
$$

and hence we can find $n_{2}=n_{2}(\epsilon)>n_{1}(\epsilon)$ such that when

$$
n>n_{2}(\epsilon), \quad 0<\epsilon<\epsilon_{1}, \text { then } \Delta_{\epsilon, n} \neq 0 .
$$

Finally, $F\left[x_{n}\right]$ is a functional of $x$ which depends only on the values of $x(t)$ at the points $t=1 / n, 2 / n, \cdots, n / n$ and is a bounded and continuous function of these values. (Here $x_{n}$ denotes the polygonalization of $x$, as defined in $\$ 1$.) We have now verified all the hypotheses of Lemma 1 for $K_{e, n}$ and the functional $F\left[x_{n}\right]$, and we therefore have when $n>n_{2}(\epsilon)$, and $0<\epsilon<\epsilon_{1}$,

$$
\int_{c}^{W} F\left[y_{n}\right] d_{W y}
$$

$$
=\left|\Delta_{\epsilon, n}\right| \int_{C}^{W} F\left[x_{n}+\int_{0}^{1} K_{\epsilon, n}(\cdot, s) x_{n}(s) d s\right] \exp \left(-\Phi_{\epsilon, n}[x]\right) d_{W} x,
$$

where

$$
\begin{aligned}
\Phi_{\epsilon, n}[x]= & \int_{0}^{1}\left[\int_{0}^{1} \frac{\partial}{\partial t} K_{\epsilon, n}(t, s) x_{n}(s) d s\right]^{2} d t \\
& +2 \int_{0}^{1}\left[\int_{0}^{1} \frac{\partial}{\partial t} K_{e, n}(t, s) x_{n}(s) d s\right] d x_{n}(t) .
\end{aligned}
$$

Our next step is to take limits as $n \rightarrow \infty$ on both sides of (10.8) and obtain

$$
\begin{aligned}
& \int_{C}^{W} F[y] d_{W} y \\
& \quad=\left|D_{c}\right| \int_{C}^{W} F\left[x+\int_{0}^{1} K_{\epsilon}(\cdot, s) x(s) d s\right] \exp \left(-\Phi_{\epsilon}(x)\right) d_{W} x,
\end{aligned}
$$

where

$$
\begin{aligned}
\Phi_{\epsilon}(x)= & \int_{0}^{1}\left[\int_{0}^{1} \frac{\partial}{\partial t} K_{\epsilon}(t, s) x(s) d s\right]^{2} d t \\
& +2 \int_{0}^{1}\left[\int_{0}^{1} \frac{\partial}{\partial t} K_{\epsilon}(t, s) x(s) d s\right] d x(t) .
\end{aligned}
$$

The passage to the limit on the left of (10.10) is immediate, since, $y_{n} \rightarrow y$ in the uniform sense and thus $F\left[y_{n}\right] \rightarrow F[y]$, and since $F\left[y_{n}\right]$ is bounded so that we may employ the principle of bounded convergence. Passing to the righthand member, we notice that (10.7) takes care of the factor outside the integral; and hence if we can show that the integrand of the right member of (10.8) approaches that of (10.10) boundedly, (10.10) will be established. 
Now since $K_{\epsilon, n}(t, s) \rightarrow K_{e}(t, s)$ uniformly in $t$ and $s$, and $x_{n}(s) \rightarrow x(s)$ uniformly in $s$, it follows that $\int_{0}^{1} K_{\epsilon, n}(t, s) x_{n}(s) d s \rightarrow \int_{0}^{1} K_{\epsilon}(t, s) x(s) d s$ uniformly in $t$ and

$$
\lim _{n \rightarrow \infty} F\left[x_{n}+\int_{0}^{1} K_{e, n}(\cdot, s) x_{n}(s) d s\right]=F\left[x+\int_{0}^{1} K_{e}(\cdot, s) x(s) d s\right]
$$

boundedly in $x$. We next show that there exist an integer $N_{0}=N_{0}(\epsilon)$ and a subset $\Gamma=\Gamma(\epsilon)$ of $C$ which is bounded in the uniform topology and such that when $x \notin \Gamma, n>N_{0}, 0<\epsilon<\epsilon_{1}$,

$$
F\left[x+\int_{0}^{1} K_{\epsilon, n}(\cdot, s) x(s) d s\right]=0 .
$$

This is based on our hypothesis that $F(x)$ vanishes outside of a bounded domain, say $G$; and on the fact that we can solve

$$
y(t)=x(t)+\int_{0}^{1} K_{\epsilon, n}(t, \xi) x(\xi) d \xi
$$

and obtain

$$
x(t)=y(t)+\int_{0}^{1} K_{\epsilon, n}^{-1}(t, \xi) y(\xi) d \xi
$$

throughout $C$ for sufficiently large $n$, since the Fredholm determinant $D_{\epsilon, n} \neq 0$ when $n>n_{1}(\epsilon), 0<\epsilon<\epsilon_{1}$. In fact, it follows from (10.6) that we can choose $N_{0}=N_{0}(\epsilon)$ so great that $\left|D_{\epsilon, n}\right| \geqq\left|D_{\epsilon}\right| / 2 \neq 0$ when $n>N_{0}$, and hence $\left[D_{\epsilon, n}\right]^{-1}$ is bounded in $n$ for all $n>N_{0}$. Thus the resolvent kernel $K_{\epsilon, n}^{-1}(t, \xi)$ is uniformly bounded in $t, \xi, n$ for fixed $\epsilon$ when $0 \leqq t \leqq 1,0 \leqq \xi \leqq 1$, $n>N_{0}(\epsilon)$, since it is the product of $\left[D_{\epsilon, n}\right]^{-1}$ by a Fredholm numerator determinant $D_{\epsilon, n}(t, s)$ which is uniformly bounded in $t, s, \epsilon, n$ because it is given by the familiar Fredholm algorithm in terms of the uniformly bounded $K_{\epsilon, n}(t, \xi)$. For fixed $\epsilon$, we now define $\Gamma=\Gamma(\epsilon)$ as the set of functions $x(t)$ obtained from (10.15) by letting $y(t)$ range over $G$ and $n$ range over the set of integers $N_{0}+1, N_{0}+2, \cdots$. We readily see that this $\Gamma$ and $N_{0}$ satisfy. the desired conditions, namely that $\Gamma$ be a subset of $C$ which is bounded in the uniform topology, and that (10.13) holds when $x \in \Gamma, n>N_{0}, 0<\epsilon<\epsilon_{1}$. Since (10.14) takes $C$ into $C$ (for $K_{\epsilon, n}(t, \xi)$ is continuous and vanishes when $t=0)$ and takes functions which are not zero at $t=0$ into functions which are not zero when $t=0$, it follows that the inverse transformation (10.15) takes $C$ into $C$, and hence $\Gamma$ is a subset of $C$. Moreover $G$ and $K_{\mathrm{e}, n}$ are uniformly bounded, and hence so is the set $\Gamma$ of functions $x(t)$ defined by (10.15) in terms of functions $y(t)$ in $G$. Finally, if $x(t) \notin \Gamma$, then for each $n>N_{0}$, the corresponding $y(t)$ defined by (10.14) is not in $G$, and since $F$ vanishes out- 
side $G, F(y)$ is zero. Thus (10.13) is established in the specified region, and the set $\Gamma$ has the specified properties.

Turning next to the second factor of the integrand of the right member of (10.8), we note that for each $x \in C, 0<\epsilon<\epsilon_{1}$,

$$
\lim _{n \rightarrow \infty} \Phi_{\epsilon, n}[x]=\Phi_{\epsilon}[x] .
$$

This follows immediately from (8.14) and (8.16) of Lemma 6, with $\partial K_{\mathbf{\epsilon}}(t, s) / \partial t$ playing the role of $H(t, s)$ of the lemma, and, in consequence, $\partial K_{\epsilon, n}(t, s) / \partial t$ playing the role of $H^{n}(t, s)$ (since (10.4) implies (8.13)). Hypotheses (8.6) and (8.7) of Lemma 6 are given by hypothesis $(0.6 \mathrm{C})$ of the theorem together with (10.1) and (10.2). Now (10.12) and (10.16) imply that for each $x$, the integrand of the right member of (10.8) approaches the integral of the right member of (10.10). It remains to show that the limits are approached boundedly. To do this, let $\gamma$ be so great that when $x(t)$ is in the uniformly bounded set $\Gamma$, we have $|x(t)| \leqq \gamma$ for all $t$ on $0 \leqq t \leqq 1$. Thus when $\max _{0 \leqq t \leqq 1}|x(t)|>\gamma, n>N_{0}, 0<\epsilon<\epsilon_{1}$, the equation (10.13) must hold; and consequently when $\max _{0 \leqq t \leqq 1}\left|x_{n}(t)\right|>\gamma, n>N_{0}, 0<\epsilon<\epsilon_{1}$, we have

$$
F\left[x_{n}+\int_{0}^{1} K_{\epsilon, n}(\cdot, s) x_{n}(s) d s\right] \exp \left(-\Phi_{\epsilon, n}(x)\right)=0 .
$$

On the other hand, if $\max _{0 \leqq t \leqq 1}\left|x_{n}(t)\right| \leqq \gamma, n>N_{0}, 0<\epsilon<\epsilon_{1}$, we find from (8.15) and (8.17) of Lemma 6 (with $\partial K_{\mathrm{c}}(t, s) / \partial t$ playing the role of $H(t, s)$ and $\gamma$ playing the role of $\mu_{n}$ ) that

$$
\left|\Phi_{\epsilon, n}(x)\right| \leqq\left[\gamma \int_{0}^{1} M_{e}(s) d s\right]^{2}+2 \gamma^{2} \int_{0}^{1}\left[M_{\epsilon}(s)+V_{\epsilon}(s)\right] d s,
$$

where $M_{\epsilon}(s)$ and $V_{\epsilon}(s)$ are the analogues of $M(s)$ and $V(s)$ of Lemma 6 as applied to the function $\partial K_{\epsilon}(t, s) / \partial t$. Thus we may take (by $(0.6 \mathrm{C}),(10.1)$, (9.9), (10.2))

$$
M_{\epsilon}(s)=\int_{0}^{1} \sup _{0 \leqq t \leqq 1}\left|\frac{\partial}{\partial t} K(t, s)\right|^{*} d s+\frac{1}{2 \epsilon} \int_{0}^{1}|J(s)| d s
$$

and

$$
V_{\epsilon}(s)=\int_{0}^{1} \operatorname{var}_{0 \leqq t \leqq 1}\left[\frac{\partial}{\partial t} K(t, s)\right]^{*} d s+\frac{2}{\epsilon} \int_{0}^{1}|J(s)| d s,
$$

where the star indicates that $\partial K / \partial t$ is to be replaced by an equivalent function for which the integrals exist, as given in (0.6C). Hence if $B$ is an upper bound for the bounded functional $F(x)$, we have when $\max _{0 \leqq \iota \geqq 1}\left|x_{n}(t)\right| \leqq \gamma$, $n>N_{0}, 0<\epsilon<\epsilon_{1}$, 


$$
\begin{aligned}
\mid F\left[x_{n}+\right. & \left.\int_{0}^{1} K_{\epsilon, n}(\cdot, s) x_{n}(s) d s\right] \exp \left(-\Phi_{\epsilon, n}(x)\right) \mid \\
& \leqq B \exp \left(\left[\gamma \int_{0}^{1} M_{\epsilon}(s) d s\right]+2 \gamma^{2} \int_{0}^{1}\left[M_{\epsilon}(s)+V_{\epsilon}(s)\right] d s\right) .
\end{aligned}
$$

Combining this with (10.17), we see that (10.21) holds whenever $n>N_{0}$, $0<\epsilon<\epsilon_{1}$ independently of the size of $\max \left|x_{n}\right|$. But the right-hand side of (10.21) is independent of $x$ and of $n$, and therefore (10.21) shows that the convergence of the integrand of the right member of (10.8) is bounded in $x$ and $n$, and the passage to the limit is justified. Using (10.12) and (10.16) in taking limits on (10.8), we obtain (10.10); and thus (10.10) together with (10.11) is established.

Having taken limits in (10.8) as $n \rightarrow \infty$ and obtained (10.10), we next take limits as $\epsilon \rightarrow 0^{+}$in (10.10) and obtain (0.15). The argument is carried out on the same lines as the preceding. The left members of $(0.15)$ and $(10.10)$ are identical, and the factor $\left|D_{\epsilon}\right|$ outside the integral on the right of $(10.10)$ approaches $|D|$ by (10.5) and we thus need only show that the integrand on the right of (10.10) approaches the integrand on the right of $(0.15)$ boundedly.

Dealing first with the factor $F$, we note that

(10.22)

$$
\begin{aligned}
\left|\int_{0}^{1} \Lambda_{\epsilon}(t, s) x(s) d s\right|= & \left|\frac{1}{2} \int_{0}^{1}\left[\Omega_{\epsilon}(s-t)-\Omega_{\epsilon}(s+0)\right] J(s) x(s) d s\right| \\
\leqq & \frac{1}{2} \int_{\max (t-\epsilon, 0)}^{\min (t+\epsilon, 1)}\left|\Omega_{\epsilon}(s-t) J(s) x(s)\right| d s \\
& +\frac{1}{2} \int_{0}^{\epsilon}\left|\Omega_{\epsilon}(s+0) J(s) x(s)\right| d s \\
\leqq & \frac{3}{2} \epsilon \max _{0 \leqq s \leqq 1}|J(s) x(s)|,
\end{aligned}
$$

so that for fixed $x$,

$$
\lim _{\epsilon \rightarrow 0^{+}} \int_{0}^{1} K_{\epsilon}(t, s) x(s) d s=\int_{0}^{1} K(t, s) x(s) d s
$$

uniformly in $t, 0 \leqq t \leqq 1$. Since $F$ is continuous in the uniform topology,

$$
\lim _{e \rightarrow 0^{+}} F\left[x+\int_{0}^{1} K_{\epsilon}(\cdot, s) x(s) d s\right]=F\left[x+\int_{0}^{1} K(\cdot, s) x(s) d s\right],
$$

and since $F$ is bounded, the limit is approached boundedly in $x$. We next show that there exist a positive number $\epsilon_{0}$ and a subset $\Gamma^{\prime}$ of $C$ which is bounded in the uniform topology and such that when $x \notin \Gamma^{\prime}, 0<\epsilon<\epsilon_{0}$, 


$$
F\left[x+\int_{0}^{1} K_{\epsilon}(\cdot, s) x(s) d s\right]=0 .
$$

Choose $G$ as before, so that $F(x)=0$ when $x \notin G$, and solve

$$
y(t)=x(t)+\int_{0}^{1} K_{\epsilon}(t, \xi) x(\xi) d \xi
$$

obtaining

$$
x(t)=y(t)+\int_{0}^{1} K_{e}^{-1}(t, \xi) y(\xi) d \xi
$$

throughout $C$ for sufficiently small $\epsilon$. By (10.5) we can choose $\epsilon_{0}$ so small that when $0<\epsilon<\epsilon_{0},\left|D_{\epsilon}\right| \geqq|D| / 2 \neq 0$, so that $\left[D_{\epsilon}\right]^{-1}$ is bounded in $\epsilon$ for all positive $\epsilon<\epsilon_{0}$. Thus the resolvent kernel $K_{\epsilon}^{-1}(t, \xi)$ is uniformly bounded in $t, \xi, \epsilon$ when $0 \leqq t \leqq 1,0 \leqq \xi \leqq 1,0<\epsilon<\epsilon_{0}$, since it is the product of $\left[D_{\epsilon}\right]^{-1}$ by a Fredholm numerator determinant $D_{\epsilon}(t, s)$ which is uniformly bounded in $t, s, \epsilon$ because it is given by the Fredholm algorithm in terms of the uniformly bounded $K_{c}(t, \xi)$. We now take $\Gamma^{\prime}$ as the set of functions $x(t)$ obtained from $(10.27)$ by letting $y(t)$ range over $G$ and $\epsilon$ range over the set $0<\epsilon<\epsilon_{0}$. Then $\Gamma^{\prime}$ and $\epsilon_{0}$ satisfy the required conditions, for $\Gamma^{\prime}$ is obviously a uniformly bounded subset of $C$, and if $x \notin \Gamma^{\prime}$, then, for each $\epsilon$ in $0<\epsilon<\epsilon_{0}$, the corresponding $y$ defined by (10.26) is not in $G$, and $F(y)=0$. Thus (10.25) is established in the specified region, and $\Gamma^{\prime}$ has the specified property.

Turning next to the second factor of the integrand of the right member of (10.10), we shall show that for each $x \in C$,

$$
\lim _{\rightarrow 0^{+}} \Phi_{6}[x]=\Phi[x] \text {. }
$$

Differentiating (10.2) and using (10.1), we have for almost all $t, s$ in the square $\left({ }^{5}\right)$,

$$
\frac{\partial}{\partial t} K_{\epsilon}(t, s)=\frac{\partial}{\partial t} K(t, s)+\frac{1}{2} \frac{\partial}{\partial t} \Omega_{\epsilon}(s-t) J(s),
$$

so that for almost all $t$ we have by (9.9),

$$
\begin{aligned}
\int_{0}^{1} \frac{\partial}{\partial t} K_{\epsilon}(t, s) x(s) d s= & \int_{0}^{1} \frac{\partial}{\partial t} K(t, s) x(s) d s \\
& +\frac{1}{2 \epsilon} \int_{\max (t-\epsilon, 0)}^{\min (t+\epsilon, 1)} J(s) x(s) d s .
\end{aligned}
$$

It follows from the law of the mean for integrals that if $\epsilon \leqq t \leqq 1-\epsilon$, the last

(๖) See footnote 2. 
term of the second number of $(10.30)$ can be written $J\left(\tau_{\epsilon, t}\right) x\left(\tau_{\epsilon, t}\right)$, where $t-\epsilon \leqq \tau_{\epsilon, t} \leqq t+\epsilon$; and hence we have (using (0.3) and (0.4)) for almost all $t$ on $0 \leqq t \leqq 1$,

$$
\begin{aligned}
\lim _{\rightarrow \rightarrow 0^{+}} \int_{0}^{1} \frac{\partial}{\partial t} K_{\epsilon}(t, s) & x(s) d s=\int_{0}^{1} \frac{\partial}{\partial t} K(t, s) x(s) d s+J(t) x(t) \\
& =\frac{d}{d t}\left[\int_{0}^{t} K^{2}(t, s) x(s) d s+\int_{t}^{1} K^{1}(t, s) x(s) d s\right] \\
& =\frac{d}{d t} \int_{0}^{1} K(t, s) x(s) d s .
\end{aligned}
$$

Moreover for fixed $x$, the limit in (10.31) is approached boundedly, since from (10.30)

$$
\begin{aligned}
& \left|\int_{0}^{1} \frac{\partial}{\partial t} K_{\epsilon}(t, s) x(s) d s\right| \\
& \quad \leqq \max _{0 \leqq s \leqq 1}|x(s)| \cdot\left\{\int_{0}^{1} \sup _{0 \leqq t \leqq 1}\left|\frac{\partial}{\partial t} K(t, s)\right| d s+\max _{0 \leqq s \leqq 1}|J(s)|\right\}
\end{aligned}
$$

and the right-hand side is finite by hypothesis $(0.6 \mathrm{C})$. Hence by the principle of bounded convergence we have from (10.31) and (10.32)

$$
\lim _{\rightarrow \rightarrow 0^{+}} \int_{0}^{1}\left[\frac{\partial}{\partial t} K_{\epsilon}(t, s) x(s) d s\right]^{2} d t=\int_{0}^{1}\left[\frac{d}{d t} \int_{0}^{1} K(t, s) x(s) d s\right]^{2} d t
$$

and we see that the first term of the second member of (10.11) approaches the first term of the right member of (0.9), and it remains to show that the second term of (10.11) approaches the sum of the second and third terms of (0.9). We do this by applying Lemma 7 to the second term of the right-hand side of (10.29), obtaining

$$
\begin{aligned}
\lim _{\epsilon \rightarrow 0} \int_{0}^{1} & {\left[\int_{0}^{1} \frac{\partial}{\partial t} K_{\epsilon}(t, s) x(s) d s\right] d x(t) } \\
& =\int_{0}^{1}\left[\int_{0}^{1} \frac{\partial}{\partial t} K(t, s) x(s) d s\right] d x(t)+\frac{1}{2} \int_{0}^{1} J(t) d[\dot{x}(t)]^{2}
\end{aligned}
$$

and thus (10.33) and (10.34) establish (10.28), which, together with (10.24), implies that the integrand on the right of (10.10) approaches the integrand on the right of $(0.15)$ for each fixed $x$.

Finally, we complete the justification of the limiting process and establish $(0.15)$ by showing that the convergence is bounded. To do this, choose $\gamma^{\prime}$ so great that when $x \in \Gamma^{\prime}, \max _{0 \leqq t \leqq 1}|x(t)| \leqq \gamma^{\prime}$, and note that when $\max _{0 \leqq t \leqq 1}|x(t)|>\gamma^{\prime}$ and $0<\epsilon<\epsilon_{0},(10.25)$ holds, so that 
(10.35)

$$
F\left[x+\int_{0}^{1} K_{\epsilon}(\cdot, s) x(s) d s\right] \exp \left(-\Phi_{\epsilon}(x)\right)=0 .
$$

On the other hand, if $\max _{0 \leqq t \leqq 1}|x(t)| \leqq \gamma^{\prime}$ and $0<\epsilon<\epsilon_{0}$, we let $B$ denote an upper bound of $F$ as before, and let $\left(^{(6)}\right.$

$$
\beta=\int_{0}^{1} \sup _{0 \leqq t \leqq 1}\left|\frac{\partial}{\partial t} K(t, s)\right| d s+\max _{0 \leqq s \leqq 1}|J(s)|
$$

so that by (10.32),

$$
\left|\int_{0}^{1}\left[\int_{0}^{1} \frac{\partial}{\partial t} K_{\epsilon}(t, s) x(s) d s\right]^{2} d t\right| \leqq\left(\gamma^{\prime} \beta\right)^{2} .
$$

Similarly, we let

$$
\beta^{\prime}=\int_{0}^{1} \operatorname{var}_{0 \leqq t \leqq 1}\left[\frac{\partial}{\partial t} K(t, s)\right] d s+\operatorname{var}_{0 \leqq s \leqq 1}[J(s)],
$$

so that by (10.29), (10.28), (9.6), (9.3), (9.4), (9.5) we have

$$
\left|\int_{0}^{1}\left[\int_{0}^{1} \frac{\partial}{\partial t} K_{\epsilon}(t, s) x(s) d s\right] d x(t)\right| \leqq\left(\gamma^{\prime}\right)^{2}\left(\beta+\beta^{\prime}\right)
$$

since

$$
\begin{aligned}
\int_{0}^{1} & {\left[\int_{0}^{1} \frac{\partial}{\partial t} K(t, s) x(s) d s\right] d x(t) } \\
& =x(1) \int_{0}^{1}\left[\frac{\partial}{\partial t} K(t, s)\right]_{t=1} x(s) \dot{d} s-\int_{0}^{1} x(t) d_{t} \int_{0}^{1} \frac{\partial}{\partial t} K(t, s) x(s) d s
\end{aligned}
$$

by integration by parts. Thus when $\max _{0 \leqq t \leqq 1}|x(t)| \leqq \gamma^{\prime}$ and $0<\epsilon<\epsilon_{0}$, we have from (10.11), (10.37), (10.39)

$$
\begin{aligned}
\left|F\left[x+\int_{0}^{1} K_{\epsilon}(\cdot, s) x(s) d s\right]\right| \exp (- & \left.\Phi_{\epsilon}(x)\right) \\
& \leqq B \exp \left(\left(\gamma^{\prime}\right)^{2}\left(\beta^{2}+2 \beta+2 \beta^{\prime}\right)\right) ;
\end{aligned}
$$

and since $(10.35)$ holds when $\max _{0 \leqq t \leqq 1}|x(t)|>\gamma^{\prime}, 0<\epsilon<\epsilon_{0}$, it follows that (10.41) holds whenever $0<\epsilon<\epsilon_{0}$, irrespective of the size of $x(t)$. But the right member of (10.41) is independent of $x$ and $\epsilon$, and therefore (10.41) shows that the convergence of the integrand of the right member of (10.10) to its limit as $\epsilon \rightarrow 0^{+}$is bounded in $x$ and $\epsilon$, and the passage to the limit is justified. Thus by (10.24) and (10.28), we obtain (0.15) from (10.10), and the proof of Theorem Ia is complete.

(8) See footnote 2 . 
11. Proof of Theorem I. We shall derive Theorem I from Theorem Ia. (The method used is similar to that used in [4] in obtaining Theorem II of that paper from Theorem $I$ of that paper.) First let $I$ be a quasi-interval

$$
\begin{gathered}
\lambda_{j}<y\left(t_{j}\right)<\mu_{j}, \\
j=1, \cdots, n ; 0<t_{1}<\cdots<t_{n} \leqq 1\left(-\infty \leqq \lambda_{j}<\mu_{j} \leqq \infty\right) .
\end{gathered}
$$

Let $\epsilon>0$ and let $\phi_{i, \epsilon}(\eta)$ be a continuous "trapezoidal" function which equals zero outside the interval $\lambda_{j}<\eta<\mu_{j}$, equals unity inside the interval $\lambda_{j}+\epsilon<\eta$ $<\mu_{j}-\epsilon$, and is linear on the remaining intervals. (If $\lambda_{j}$ takes on the improper value $-\infty$, so does $\lambda_{j}+\epsilon$, and so on.)

Also let $\psi_{\epsilon}(u)$ be a continuous polygonal function on $0 \leqq u<\infty$ which equals zero for $u>1+1 / \epsilon$, equals unity for $0 \leqq u<1 / \epsilon$ and is linear elsewhere.

Let $p_{I}(y)$ be the characteristic functional of $I$ (that is, $p_{I}(y)=1$ if $y \in I$ and 0 otherwise), and let

$$
p_{I, \epsilon}(y)=\psi_{\epsilon}\left[\max _{0 \leqq t \leqq 1}|y(t)|\right] \prod_{j=1}^{n} \phi_{j, \epsilon}\left(y\left(t_{j}\right)\right) .
$$

First we observe that

$$
p_{I, \epsilon}(y) \nearrow p_{I}(y) \text { as } \epsilon \searrow 0
$$

$(y(\cdot)$ in $C)$,

for if $y(\cdot) \notin I$, then at least one of the inequalities (11.1) is not satisfied and the corresponding $\phi_{i, \epsilon}\left(y\left(t_{j}\right)\right)$ will be zero for every $\epsilon>0$, while if $y(\cdot) \in I$, then for sufficiently small positive $\epsilon$ each factor $\phi_{j, \epsilon}\left(y\left(t_{j}\right)\right)$ will be unity and also the factor $\psi_{\mathrm{e}}[\max |y(t)|]$ will be unity. Thus (11.3) holds for all $y$ on $C$. Next we note that

$$
F[y] \equiv p_{I, \epsilon}[y]
$$

satisfies the hypotheses of Theorem Ia. (Boundedness, Wiener summability and the fact that it vanishes outside a uniformly bounded domain are obvious. Continuity follows from the continuity of the individual factors in (11.2).) Hence the conclusion (0.15) of Theorem Ia holds for $p_{I, \epsilon}[y]$, and by monotone convergence (see (11.3)) it also holds for $p_{I}[y]$.

This yields

(11.4) $\int_{C}^{W} p_{I}[y] d_{W} y=|D| \int_{C}^{W} p_{I}\left[x+\int_{0}^{1} K(\cdot, s) x(s) d s\right] \exp (-\Phi[x]) d_{W} x$

which is equivalent to (0.8) of Theorem I with $S=T^{-1} I$. By progressively enlarging the scope of $S$ we obtain (0.8) for every Wiener measurable subset $S$ of $C$. The details of the proof of this and of the proof of the remaining portion of Theorem I are entirely similar to those given in $\$ 5$ of [4]; for this reason we omit further details of the proof. 
12. Proof of Theorem II. We now consider the transformation $L$ of $(0.11)$, and we resolve it into the product of two transformations

$$
\begin{aligned}
T: z(t) & =x(t)+\int_{0}^{1} K(t, s) x(s) d s, \\
T^{\prime}: & y(t)=z(t)+x_{0}(t) .
\end{aligned}
$$

The transformation $T$ is one to which Theorem I applies, and $T^{\prime}$ is one to which Theorem II of [4] applies $\left({ }^{7}\right)$. Hence if $S$ is any Wiener measurable subset of $C$ and if $F[y]$ is any Wiener measurable functional defined for $y$ in $T^{\prime} T S$, then by Theorem II of [4] we have

$$
\begin{aligned}
& \int_{T^{\prime} T S}^{W} F[y] d_{W} y \\
= & \exp \left(-\int_{0}^{1}\left[x_{0}^{\prime}(t)\right]^{2} d t\right) \int_{T S}^{W} F\left[z+x_{0}\right] \exp \left(-2 \int_{0}^{1} x_{0}^{\prime}(t) d z(t)\right) d_{W},
\end{aligned}
$$

this equality holding whenever either member exists.

Next we apply the transformation $T$ using the (measurable) functional

$$
G[z]=F\left[z+x_{0}\right] \exp \left(-2 \int_{0}^{1} x_{0}^{\prime}(t) d z(t)\right) ;
$$

we find

$$
\begin{aligned}
& \exp \left(-\int_{0}^{1}\left[x_{0}^{\prime}(t)\right]^{2} d t\right) \int_{T S}^{W} F\left[z+x_{0}\right] \exp \left(-2 \int_{0}^{1} x_{0}^{\prime}(t) d z(t)\right) d_{W} z \\
& =|D| \exp \left(-\int_{0}^{1}\left[x_{0}^{\prime}(t)\right]^{2} d t\right) \int_{S}^{W} F\left[x+x_{0}+\int_{0}^{1} K(\cdot, s) x(s) d s\right]
\end{aligned}
$$

$$
\begin{aligned}
& \cdot \exp \left(-2 \int_{0}^{1} x_{0}^{\prime}(t) d x(t)\right) \\
& \cdot \exp \left(-2 \int_{0}^{1} x_{0}^{\prime}(t) d_{t}\left[\int_{0}^{1} K(t, s) x(s) d s\right]\right) \exp (-\Phi[x]) d_{W} x \\
& =|D| \int_{S}^{W} F\left[x+x_{0}+\int_{0}^{1} K(\cdot, s) x(s) d s\right] \exp (-\Psi[x]) d_{W} x
\end{aligned}
$$

$(7)$ In the translation theorems of [4] it was actually assumed that $x_{0}^{\prime}(t)$ exists everywhere on $0 \leqq t \leqq 1$ and that $x_{0}^{\prime}(t)$ is of bounded variation on $0 \leqq t \leqq 1$. It is easily seen that the two theorems of [4] still hold if it is assumed merely that $x_{0}(t)$ is absolutely continuous on $0 \leqq t \leqq 1$ and that $x_{0}^{\prime}(t)$ is equivalent to a function $w(t)$ which is of bounded variation on $0 \leqq t \leqq 1$. It is this slightly modified form of Theorem II of [4] which applies to the transformation (12.2) of the present paper. 
Combining (12.3) and (12.5) we obtain the conclusion (0.14) of Theorem II.

By selecting $F[y]$ to be the characteristic functional of the set $L S$ we obtain (0.13).

13. An example. In this section we use our transformation theorem, Theorem I, to evaluate the characteristic function

$$
\int_{C}^{W} \exp \left(i \mu \int_{0}^{1}[x(t)]^{2} d t\right) d_{W} x \quad(-\infty<\mu<\infty)
$$

of the chance variable $\int_{0}^{1}[x(t)]^{2} d t$. The characteristic function (13.1) is evaluated ( 8 ) by a consideration of the special linear transformation

$$
T: y(t)=x(t)+\lambda \int_{0}^{t} \tan \lambda(s-1) \cdot x(s) d s
$$

for

$$
-\pi / 2<\lambda<\pi / 2 \text {. }
$$

We shall first show that the transformation (13.2) satisfies the hypotheses of Theorem I. Writing

$$
\begin{aligned}
& K^{1}(t, s)=0 \\
& K^{2}(t s)=\lambda \tan \lambda(s-1) \\
& \text { (13.5) } K(t, s)=\left\{\begin{array}{l}
0 \\
\lambda \tan \lambda(s-1) \\
2^{-1} \lambda \tan \lambda(s-1)
\end{array}\right. \\
& \text { when } 0 \leqq t<s, 0<s \leqq 1 \text {, } \\
& J(s)=2^{-1} \lambda \tan \lambda(s-1),
\end{aligned}
$$$$
(0 \leqq t \leqq 1,0 \leqq s \leqq 1),
$$$$
\text { when } s<t<1,0 \leqq s \leqq 1 \text {, }
$$$$
\text { when } t=s, 0 \leqq s \leqq 1 \text {, }
$$$$
0 \leqq s \leqq 1 \text {, }
$$

we easily see that (0.6 A-D) are satisfied. Also

$$
\begin{aligned}
& D=1+\sum_{p=1}^{\infty} \frac{1}{p !} \int_{0}^{1} \cdots \int_{0}^{1}\left|\begin{array}{c}
K\left(s_{1}, s_{1}\right) \cdots K\left(s_{1}, s_{p}\right) \\
. \cdots \cdots \\
\dot{K\left(s_{p}, s_{1}\right)} \cdots \cdot K\left(s_{p}, s_{p}\right)
\end{array}\right| d s_{1} \cdots d s_{p} \\
& =\sum_{p=0}^{\infty} \frac{1}{p !}\left\{\int_{0}^{1} K(s, s) d s\right\}^{p}=\exp \left(\int_{0}^{1} K(s, s) d s\right) \\
& =\exp \left(\frac{1}{2} \int_{0}^{1} \lambda \tan \lambda(s-1) d s\right)=\exp \left(\left.\frac{1}{2} \log \sec \lambda(s-1)\right|_{0} ^{1}\right) \\
& =\exp \left(-\frac{1}{2} \log \sec \lambda\right)=(\cos \lambda)^{1 / 2} .
\end{aligned}
$$

(8) The expression (13.1) can also be evaluated by a modification of the method of Paley and Wiener [2] using the statistical independence of terms in the orthogonal development of $d x(t)$. For this method it seems preferable (or even necessary) to use as an orthogonal set the set $\left\{2^{1 / 2} \cos (n+1 / 2) \pi t\right\}$, rather than the set $\{\exp (2 n \pi i t)\}$. 
Thus $D \neq 0$. Hence $(0.6 \mathrm{E})$ holds and Theorem I applies to the transformation (13.2). We apply the transformation formula $(0.10)$ to the entire space $C$ :

$$
\begin{gathered}
\int_{C}^{W} F[y] d_{W} y=|D| \int_{C}^{W} F\left[x+\lambda \int_{0}^{t} \tan \lambda(s-1) \cdot x(s) d s\right] \\
\cdot \exp (-\Phi[x]) d_{W} x
\end{gathered}
$$

with

$$
|D|=(\cos \lambda)^{1 / 2}
$$

and

$$
\begin{aligned}
\Phi[x] & =\int_{0}^{1} \lambda^{2} \tan ^{2} \lambda(s-1) \cdot[x(s)]^{2} d s+\int_{0}^{1} \lambda \tan \lambda(s-1) d\left\{[x(s)]^{2}\right\} \\
& =\lambda^{2} \int_{0}^{1} \tan ^{2} \lambda(s-1) \cdot[x(s)]^{2} d s-\lambda^{2} \int_{0}^{1}[x(s)]^{2} \sec ^{2} \lambda(s-1) d s \\
& =-\lambda^{2} \int_{0}^{1}[x(s)]^{2} d s
\end{aligned}
$$

Hence

$$
\begin{gathered}
\int_{C}^{W} F[y] d_{W} y=(\cos \lambda)^{1 / 2} \int_{C}^{W} F\left[x+\lambda \int_{0}^{t} \tan \lambda(s-1) \cdot x(s) d s\right] \\
\cdot \exp \left(\lambda^{2} \int_{0}^{1}[x(s)]^{2} d s\right) d_{W} x
\end{gathered}
$$

and with $F[y] \equiv 1$ we obtain

$$
\int_{C}^{W} \exp \left(\lambda^{2} \int_{0}^{1}[x(s)]^{2} d s\right) d_{W} x=\frac{1}{(\cos \lambda)^{1 / 2}} .
$$

The relation (13.12) holds for all real $\lambda$ in $-\pi / 2<\lambda<\pi / 2$, since the transformation (13.2) meets the requirements of our theorem for such values of $\lambda$. The right-hand member of equation (13.12) is single-valued and analytic in the complex $\lambda$-plane if this plane is slit along the real axis from $(\pi / 2, \infty)$ and from $(-\pi / 2,-\infty)$. (We consider the branch which is positive for $\lambda$ real and in $-\pi / 2<\lambda<\pi / 2$.) With this interpretation equation (13.12) holds for all complex values of $\lambda$ for which the integral in (13.12) converges and represents an analytic function. Now the integral in (13.12) converges for all real values of $\lambda$ in $\lambda^{2}<\pi^{2} / 4$. Also if $\lambda$ is complex we have

$$
\int_{C}^{W}\left|\exp \left(\lambda^{2} \int_{0}^{1}[x(s)]^{2} d s\right)\right| d_{W} x=\int_{C}^{W} \exp \left(\left(\operatorname{Re} \lambda^{2}\right) \int_{0}^{1}[x(s)]^{2} d s\right) d_{W} x,
$$


and hence the integral in (13.12) converges for $\operatorname{Re} \lambda^{2}<\pi^{2} / 4$. If we consider the left member of (13.12) as a function of the complex variable $\lambda^{2}$ and integrate around a contour lying in $\operatorname{Re} \lambda^{2}<\pi^{2} / 4$, we are able to interchange the order of integration by the (mixed) Fubini theorem. Hence, by Morera's theorem, the left member of (13.12) is analytic in $\operatorname{Re} \lambda^{2}<\pi^{2} / 4$, and hence (13.12) holds for $\operatorname{Re} \lambda^{2}<\pi^{2} / 4$, with $(\cos \lambda)^{1 / 2}$ having the determination which is real and positive for $\lambda$ real and on the interval $-\pi / 2<\lambda<\pi / 2$. (It may be noted that the Wiener integral in (13.12) diverges for $\operatorname{Re} \lambda^{2} \geqq \pi^{2} / 4$ and hence (13.12) holds whenever the integral has a meaning.) Writing $\lambda^{2}=i \mu$, where $\mu$ is real, we obtain the result (0.2) mentioned in the introduction.

\section{BIBLIOGRAPHY}

1. N. Wiener, Generalized harmonic analysis, Acta Math. vol. 55 (1930) pp. 117-258, especially pp. 214-234.

2. R. E. A. C. Paley and N. Wiener, Fourier transforms in the complex domain, Amer. Math. Soc. Colloquium Publications, vol. 19, New York, 1934, see especially chaps. 9 and 10.

3. R. H. Cameron and W. T. Martin, An expression for the solution of a general class of non-linear integral equations, Amer. J. Math. vol. 66 (1944) pp. 281-298.

4. - On transformations of Wiener integrals under translations, Ann. of Math. vol. 45 (1944) pp. 386-396.

The Massachusetts Institute of Technology,

Cambridge, Mass.

SYRACUSE UNIVERSITY,

Syracuse, N. Y. 